\title{
Dilepton production in heavy-ion collisions with in-medium spectral functions of vector mesons
}

\author{
E. Santini ${ }^{a}$, M.D. Cozma ${ }^{a, b}$, Amand Faessler $^{a}$, C. Fuchs $^{a}$, \\ M. I. Krivoruchenko ${ }^{a, c}$, B. Martemyanov ${ }^{a, c}$ \\ ${ }^{a}$ Institut für Theoretische Physik, Tübingen Universität, Auf der Morgenstelle 14 \\ D-72076 Tübingen, Germany \\ ${ }^{b}$ National Institute for Physics and Nuclear Engineering, Atomistilor 407 \\ 077125 Magurele-Bucharest, Romania and \\ ${ }^{c}$ Institute for Theoretical and Experimental Physics, B. Cheremushkinskaya 25 \\ 117259 Moscow, Russia
}

\begin{abstract}
The in-medium spectral functions of $\rho$ and $\omega$ mesons and the broadening of nucleon resonances at finite baryon density are calculated self-consistently by combining a resonance dominance model for the vector meson production with an extended vector meson dominance model. The influence of the in-medium modifications of the vector meson properties on the dilepton spectrum in heavy-ion collisions is investigated. The dilepton spectrum is generated for the $\mathrm{C}+\mathrm{C}$ reaction at $2.0 \mathrm{~A} \mathrm{GeV}$ and compared with recent HADES Collaboration data. The collision dynamics is then described by the Tübingen relativistic quantum molecular dynamics transport model. We find that an iterative calculation of the vector meson spectral functions that takes into account the broadening of the nucleon resonances due to their increased in-medium decay branchings is convergent and provides a reasonable description of the experimental data in the mass region $0.45 \leq M \leq 0.75 \mathrm{GeV}$. On the other side, the theoretical calculations slightly underestimate the region $m_{\pi} \leq M \leq 0.4 \mathrm{GeV}$. Popular in-medium scenarios such as a schematic collisional broadening and dropping vector mesons masses are discussed as well.

PACS numbers: $12.40 \mathrm{Vv}, 25.75 \mathrm{Dw}$
\end{abstract}

\section{INTRODUCTION}

It is a well-established fact that hadrons change their properties in a dense and excited nuclear medium. Such changes are reflected in mass shifts and/or in the development of complex spectral properties. A typical example is the nucleon that suffers a substantial mass shift at finite density, see, e.g., Ref. [1], but maintains its good quasiparticle properties. Besides a shift of the pole mass, resonances, both nucleonic and mesonic, have the tendency to be broadened and to develop spectral distributions that may even lead to a loss of good quasiparticle properties. For example, total photoabsorption cross sections on heavy nuclei [2, 3] provide evidence for a substantial collisional broadening or melting of nucleon resonances inside the medium [4].

To study the medium modifications of hadrons is of particular interest, since it not only provides insight into the properties of the strongly interacting hadronic many-body systems but also allows conclusions to be drawn on QCD "observables" that characterize the medium. A prominent example is the scalar quark condensate $\langle\bar{q} q\rangle$ which determines the chiral symmetry breaking scale of QCD in the nonperturbative sector.

Heavy-ion reactions present therefore a unique opportunity for the study of nuclear or hadronic matter under extreme conditions, i.e., at supranormal densities and high temperatures. Photoor hadron-induced reactions on the nucleus provide complementary information on cold matter at 
moderate densities.

The light vector mesons $\rho$ and $\omega$ are both of particular interest, because their decay into dileptons allows one to probe the electromagnetic response of the medium. For this purpose, electromagnetic probes such as dilepton pairs have proven to be most efficient, since they leave the medium essentially undistorted by final-state interactions. In heavy-ion reactions, they provide a clear view of the effective degrees of freedom at high baryon density and temperature.

Theoretically, an abundance of models can predict the changes of vector meson masses and widths in high density, high temperature nuclear matter, and they can be roughly divided in four different classes: Brown-Rho scaling [5], models based on QCD sum rules [6, 7, 8, 9, 10], dispersion relations [10, 11, 12, 13], and effective hadronic models [10, 14, 15, 16, 17, 18, 19, 20, 21, 22, 23]. The first approaches to the description of the in-medium vector mesons were based on effective field theories (EFTs) 24] and the Nambu-Jona-Lasinio (NJL) model [25]. In some aspects, the various approaches come to qualitatively similar conclusions; however, the overall situation is still unclear. While Brown-Rho scaling, at least in its naive form, predicts a common downward mass shift of the vector mesons where the quasiparticle properties are essentially maintained, the hadronic models come to different conclusions. Concerning the $\rho$ meson, these sets of models predict in general a significant broadening of the $\rho$ and the development of complex structures in the spectral functions, e.g., the appearance of additional peaks caused by the coupling to nucleon resonances. In some cases, this occurs in line with a slight shift of the quasiparticle peak which corresponds to an additional mass shift [10]. Concerning the $\omega$ meson, the situation is even less clear. Early QCD sum rules calculations predicted even a repulsive mass shift [6], while in Refs. [8, 9] the strong dependence of the $\omega$ properties on the higher order unknown quark condensates has been pointed out, which leaves room for mass shifts in both directions. The hadronic approaches predict in common an essential broadening of the $\omega$, although they range from a strong downward mass shift [10] to a slight upward mass shift [20, 23] to an essential repulsive mass shift [19].

However, recent progress from the experimental side allows one, at least partially, to constrain the various theoretical models. While the CERES [26, 27] and HELIOS [28] dilepton experiments at the CERN Super Proton Synchroton (SPS) revealed clear evidence for in-medium effects in heavy-ion reactions $(\mathrm{Pb}+\mathrm{Au})$ through the observed enhancement of the dilepton spectra below the the $\rho$ and $\omega$ peaks relative to standard hadronic cocktail sources, such a behavior could be explained either within a scenario of a dropping $\rho$ vector meson mass [29] or by the inclusion of in-medium spectral functions for the vector mesons [17]. Thanks to unprecedented resolution, the recent NA60 dimuon experiment [30] was able to "measure" the in-medium $\rho$ spectral function under the conditions of ultrarelativistic heavy-ion collisions. NA60 seems to rule out a naive dropping mass scenario but supports the picture of modified $\rho-\omega$ spectral functions predicted by hadronic many-body theory [31].

A second set of heavy-ion experiments have been performed at laboratory energies of $1.0 \mathrm{~A} \mathrm{GeV}$ $(\mathrm{Ca}+\mathrm{Ca}$ and $\mathrm{C}+\mathrm{C})$ by the DLS Collaboration at the LBNL Bevelac [32, 33]. Also in this case, the low mass region of the dilepton spectra is underestimated by present transport calculations, in contrast to similar measurements (1.04-4.88 GeV/nucleon) for the $p+p$ and $p+d$ systems. As opposed to the ultrarelativistic case, the situation does not improve when the in-medium spectral functions or the dropping mass scenarios are taken into account [34, 35] (the DLS puzzle). Other scenarios such as possible contributions from the quark-gluon plasma or in-medium modifications of the $\eta$ mass have been excluded as a possible resolution of this puzzle. Decoherence effects [36] 
have proven to be partially successful in explaining the difference between the DLS data and the theoretical predictions. However, in this energy regime, which probes the high density, low temperature phase, the situation is going to be improved significantly with the already existing and forthcoming measurements of the HADES Collaboration at GSI [37, 38, 39]. Complemented are the heavy-ion experiments by $\gamma$-nucleus reactions. The CB-TAPS experiment [40], which focused exclusively on the $\omega$ meson and reported an enhanced strength below the $\omega$ peak, reports a broadening of the $\omega$ observed in $\gamma$-nucleus reactions. Also, the dilepton mass spectrum measured at Japan's National Laboratory for High Energy Physics (KEK) in $p+A$ reactions at a beam energy of $12 \mathrm{GeV}$ [41, 42] revealed an excess of the dileptons below the $\rho$-meson peak over known sources. However, these data could not be explained within the standard dropping mass scenario and/or assuming a significant collision broadening of the vector mesons [43]. An enhanced bremsstrahlung contribution, which is presently under debate at low energies [44, 45], will most likely not help explain the high energy KEK data.

A major difficulty in the interpretation of heavy-ion collision experimental data lies in the fact that the gap between observables and theoretically predicted in-medium properties of hadrons has to be filled by transport models. Transport models account for complicated reaction dynamics and provide the link between theory and experiment. A drawback of such a procedure is dependence on the phenomenology and an extended set of input parameters entering the models. Usually, a significant fraction of such parameters can be neither constrained by data nor based on well-established theoretical approaches. This certainly diminishes the possibility of testing experimentally theory and drawing physical conclusions from the experimental data.

In the present work, we remove some model uncertainties by applying a unified description of vector meson production, vacuum decays, and in-medium properties of vector mesons. For this purpose, we use a resonance dominance model for nucleon-nucleon scattering in combination with an extended vector meson dominance (eVMD) model. Nucleon resonance dominance (NRD) is an effective principle which assumes that vector meson production runs over the excitation of nucleon resonances [20, 46, 47, 48]. On the other hand, eVMD introduces radially excited $\rho$ and $\omega$ mesons [49] in the $R N \gamma$ transition form factors [46] in order to fulfill the quark counting rules as a strict consequence of QCD [50]. This allows the kinematically complete, gauge invariant, fully relativistic, and unified description of the nucleon resonance transition amplitudes $R \rightarrow N V$ $(V=\omega, \rho), R \rightarrow N \gamma, \gamma^{*} N \rightarrow R$ (electro-production), and $R \rightarrow N e^{+} e^{-}$with arbitrary spin and parity in terms of the magnetic, electric, and Coulomb transition form factors. The eVMD model solves a long-standing problem of VMD which underestimates the $\rho$-meson branchings of nucleon resonances when the normalization to the photon branchings is performed. The parameters of eVMD are fixed by fitting to photoproducion and electroproduction experimental data, by using results of the $\pi N$ multichannel partial-wave analysis, and, when the experimental data are not available, by using predictions of the quark models [46].

Once the model parameters are fixed, one obtains a unified (and parameter free) description of quite a broad range of physical processes including vector meson decays, nucleon resonance decays to vector mesons and dileptons, and vector meson and dilepton production in elementary and heavy-ion reactions. The NRD+eVMD model has successfully been applied earlier to vector meson $(\omega$ and $\phi)$ production in elementary $(p+p)$ reactions [51, 52] and dilepton production in elementary $p+p$ and $p+d$ reactions [47]. In Ref. [53] it has been further demonstrated that this model is qualitatively able to explain the $\omega$ and $\phi$ angular distributions in $p+p$ reactions [54, 55]. 
Embedded within the framework of the Tübingen relativistic quantum molecular dynamics (RQMD) transport model [56, 57], the NRD+eVMD model has been applied to heavy-ion reactions without introducing new parameters [36, 58]. The comparison with the dilepton data from DLS [32, 33] and HADES [38] collaborations revealed clear evidence for the in-medium effects required, in particular, to suppress excessive dilepton production from the $\omega$-meson decays. In Refs. [36, 58] the collisional broadening and dropping the vector meson masses have been analyzed phenomenologically. In the present work, we go beyond the phenomenological analysis by calculating the in-medium spectral functions of the $\rho$ and $\omega$ mesons and nucleon resonances using the NRD+eVMD approach. This allows the first self-consistent theoretical description to be made of dilepton spectra based on a unified model for nucleon resonances, vector mesons, and dilepton production, and their in-medium modifications.

\section{IN-MEDIUM SPECTRAL FUNCTIONS}

\section{A. Resonance model}

The in-medium properties of hadrons are generally expressed in terms of the self-energy $\Sigma_{V}$. The self-energy determines the spectral function of the quasiparticle in the medium. As long as the self-energy shows only a moderate energy dependence, the real part of $\Sigma_{V}$ can be interpreted in terms of a mass shift, while the imaginary part generates the in-medium width. To leading order in density, the self-energy is determined by the forward scattering length of the hadron with the surrounding particles. Since the $\rho$-nucleon and $\omega$-nucleon scattering lengths are unknown from the experimental side, these quantities have to be determined theoretically.

In the present work, we apply the resonance model to calculate the forward scattering of vector mesons on nucleons. The resonance model is not a field theory in the strict sense where corresponding Feynman diagrams are evaluated but rather an effective model that has some similarity to a field theory based on Feynman diagrams with the intermediate resonances in the $s$ channel of vector meson and nucleon scattering. Such an approach was applied in many previous investigations of vector mesons properties in the nuclear medium [13, 20, 21, 59, 60]. The present approach differs with respect to previous investigations by the fact that in the NRD+eVMD model the corresponding couplings of resonances to the nucleon and vector meson are of relativistic form and kinematically complete.

The self-energy $\Sigma_{V}$ of a vector meson $V$ in an isotopically symmetric nuclear medium is determined by the invariant $V N$ forward scattering amplitude $A_{V N}$

$$
\Sigma_{V}=-\int A_{V N} 2 \times 2 \frac{d^{3} p_{N}}{2 E_{N}(2 \pi)^{3}} .
$$

Here $V$ refers either to a $\rho^{0}$ or a $\omega$ meson. Due to isosymmetry of the medium, the self-energy $\Sigma_{V}$ for $\rho^{ \pm}$mesons is the same as for $\rho^{0}$ meson. The forward scattering amplitude $A_{V N}$ is the same for proton $(N=p)$ and neutron $(N=n)$ scattering. The integral in Eq. (1) runs over the nucleon momenta within the Fermi sphere with Fermi momentum determined by nuclear matter density $\rho_{B}$

$$
\rho_{B}=\frac{2}{3 \pi^{2}} p_{F}^{3} .
$$


The amplitude $A_{V N}$ is of Breit-Wigner form for resonance scattering

$$
A_{V N}=-\sum_{R} \frac{\left(2 J_{R}+1\right)}{2 \times 3} \frac{8 \pi s}{k} \frac{\Gamma_{R N V}(s)}{s-M_{R}^{2}+i \sqrt{s} \Gamma_{R}^{\mathrm{tot}}(s)} .
$$

In Eq. (3) the scattered vector meson has running mass squared $M^{2}$ and momentum $p, s=\left(p_{N}+p\right)^{2}$ is the running mass squared of the baryon resonance $R$, and $k$ is the c.m. momentum. The width $\Gamma_{R N V}(s)$ refers to the decay of the baryon resonance $R$ to nucleon $N$ and vector meson $V$ with fixed mass squared $M^{2}$.

The width $\Gamma_{R}^{\text {tot }}(s)$ refers to the decays of resonance $R$ not modified by the medium, in particular, with the vacuum spectral functions for the decay products. This represents the first approximation in the calculation of the medium contribution $\Sigma_{V}$ to the total self-energy $\Sigma_{V}^{\text {tot }}=\Sigma_{V}+\Sigma_{V}^{(0)}$ of the vector meson $V$. The vacuum self-energy $\Sigma_{V}^{(0)}$ is determined by the corresponding vacuum width

$$
\Im \Sigma_{V}^{(0)}=-m_{V} \Gamma_{V}^{\mathrm{tot}}(M), \Re \Sigma_{V}^{(0)}=0 .
$$

Here $\Gamma_{\rho}^{\text {tot }}(M), \Gamma_{\omega}^{\text {tot }}(M)$ are essentially given by the decay widths of the $\rho$ meson into two pions and of the $\omega$ meson into three pions, respectively. The two-pion decay width of the $\rho$ meson is given by

$$
\Gamma_{\rho}^{\mathrm{tot}}(M)=\Gamma_{\rho}^{\mathrm{tot}}\left(m_{\rho}\right) \frac{m_{\rho}}{M}\left(\frac{k_{\pi}\left(M, m_{\pi}, m_{\pi}\right)}{k_{\pi}\left(m_{\rho}, m_{\pi}, m_{\pi}\right)}\right)^{3} \Theta\left(M^{2}-4 m_{\pi}^{2}\right)
$$

where $k_{\pi}\left(M, m_{\pi}, m_{\pi}\right)$ is the momentum of the pions in the rest frame of the decaying $\rho$ meson having mass $M ; m_{\rho}$ is the physical $\rho$ meson mass and $\Gamma_{\rho}^{\text {tot }}\left(m_{\rho}\right)=150 \mathrm{MeV}$ the on-shell decay width. The three-pion decay width of the $\omega$ meson can be calculated according to the two-step process $\omega \rightarrow \rho \pi \rightarrow 3 \pi$ as proposed by Gell-Mann, Sharp, and Wagner [61]. The corresponding result can be parametrized in the simple form

$$
\Gamma_{\omega}^{\mathrm{tot}}(M)=\Gamma_{\omega}^{\mathrm{tot}}\left(m_{\omega}\right) \frac{m_{\omega}}{M}\left(\frac{M^{2}-9 m_{\pi}^{2}}{m_{\omega}^{2}-9 m_{\pi}^{2}}\right)^{3} \Theta\left(M^{2}-9 m_{\pi}^{2}\right)
$$

with $m_{\omega}$ the physical $\omega$-meson mass, and $\Gamma_{\omega}^{\mathrm{tot}}\left(m_{\omega}\right)=8.4 \mathrm{MeV}$ the on-shell decay width.

In the next order, the medium modification of the resonance spectral function including the modification of the resonance width due to the modifications of products of the resonance decay should be taken into account.

The width $\Gamma_{R N V}(s)$ can be expressed by the helicity amplitudes $A_{\frac{3}{2}}=<1-\frac{1}{2}|S| \frac{3}{2}>, A_{\frac{1}{2}}=<$ $1 \frac{1}{2}|S| \frac{1}{2}>, S_{\frac{1}{2}}=<0-\frac{1}{2}|S| \frac{1}{2}>$ of the $R \rightarrow N V$ decay [46]

$$
\Gamma_{R N V}(s)=\frac{k}{8 \pi s} \frac{2\left(A_{\frac{3}{2}}^{2}+A_{\frac{1}{2}}^{2}+S_{\frac{1}{2}}^{2}\right)}{\left(2 J_{R}+1\right)} .
$$

The calculation of these amplitudes uses the coupling constants of vector mesons to the $R N$ transition current. They were obtained in Ref. [46] by fitting photoproduction and electroproduction amplitudes of baryonic resonances in the eVMD model. The transverse and longitudinal self-energies $\Sigma_{V}^{T}$ and $\Sigma_{V}^{L}$ can be obtained by the following substitutions in Eq. (7):

$$
\begin{aligned}
& \frac{2}{3}\left(A_{\frac{3}{2}}^{2}+A_{\frac{1}{2}}^{2}+S_{\frac{1}{2}}^{2}\right) \rightarrow\left(A_{\frac{3}{2}}^{2}+A_{\frac{1}{2}}^{2}\right) \frac{1+\cos ^{2} \theta}{2}+2 S_{\frac{1}{2}}^{2} \frac{\sin ^{2} \theta}{2}, \\
& \frac{2}{3}\left(A_{\frac{3}{2}}^{2}+A_{\frac{1}{2}}^{2}+S_{\frac{1}{2}}^{2}\right) \rightarrow 2 S_{\frac{1}{2}}^{2} \cos ^{2} \theta+\left(A_{\frac{3}{2}}^{2}+A_{\frac{1}{2}}^{2}\right) \sin ^{2} \theta,
\end{aligned}
$$


where $\theta$ is the polar angle of vector meson momentum in the c.m. system. The polarization averaged self-energy $\Sigma_{V}$ reads then

$$
\Sigma_{V}=\frac{2 \Sigma_{V}^{T}+\Sigma_{V}^{L}}{3}
$$

The vector meson spectral function $\mathcal{A}_{V}$ is defined by the off-shell self-energy $\Sigma_{V}^{\text {tot }}(M,|\mathbf{p}|)$ as follows

$$
\mathcal{A}_{V}(M,|\mathbf{p}|)=\frac{1}{\pi} \frac{-\Im \Sigma_{V}^{\mathrm{tot}}}{\left(M^{2}-m_{V}^{2}-\Re \Sigma_{V}^{\mathrm{tot}}\right)^{2}+\left(\Im \Sigma_{V}^{\mathrm{tot}}\right)^{2}} .
$$

The helicity amplitudes entering into Eq. (7) have been calculated within the same relativistic approach [46] and with the same set of baryonic resonances $R$ that has successfully been applied to dilepton and vector meson production in $p+p$ collisions [47, 51, 52]. This includes the following set of resonances for $\rho N$ and $\omega N$ scattering: $\quad N^{*}(1535) \frac{1}{2}^{-}, \quad N^{*}(1650) \frac{1}{2}^{-}, \quad N^{*}(1520) \frac{3}{2}^{-}, \quad N^{*}(1440) \frac{1}{2}^{+}, \quad N^{*}(1720) \frac{3}{2}^{+}, \quad N^{*}(1680) \frac{5}{2}^{+}$, $\Delta(1620) \frac{1}{2}^{-}, \Delta(1700) \frac{3}{2}^{-}, \Delta(1232) \frac{3}{2}^{+}, \Delta(1905) \frac{5}{2}^{+}, \Delta(1950) \frac{7}{2}^{+}$.

A straightforward extension of the approach to finite temperature and baryon chemical potential would be to integrate the present amplitudes, Eq. (7), over hot Fermi distributions. This can easily be done and will be a first step toward an application, e.g., at SPS conditions. However, for a meaningful determination of spectral functions at SPS conditions, one would have to take into account the coupling to not only baryonic but also mesonic excitations $(\pi, K, \ldots)$ 31].

So far, analyticity has not been used in the data analyses to determine the multichannel $\pi N$ scattering amplitudes [62, 63, 64]. The current phenomenological schemes provide resonance masses and widths, based on multichannel unitarity and other, less fundamental constraints. The background phases entering the dispersion relations are not provided.

We did not attempt to embed analyticity and restricted our approach to energies $s<4 \mathrm{GeV}^{2}$, where the sum over Breit-Wigner poles gives typically a good approximation for the amplitudes 65]. The background is described by $t$-channel $\sigma$-meson exchange and the $u$-channel part of the Compton $\rho N$ scattering diagram evaluated in the Born approximation.

\section{B. Nonresonant contributions}

Up to now we have not discussed possible nonresonant contributions to the forward vector meson-nucleon scattering. The reason is twofold. First, we cannot fix the nonresonant amplitudes with the same accuracy as the resonant ones. Second, if we fix them with the available accuracy we would find that nonresonant amplitudes approximately cancel in the sum. For example, in the case of the $\rho$ meson, there exist the Compton scattering amplitude which gives a positive contribution to the real part of the $\rho$-meson self-energy and the amplitude due to $\sigma$-meson exchange which gives a negative contribution to it (the latter is of the same origin as the attractive part of the $N N$ interaction [66]). The unknown $\rho \rho \sigma$ coupling constant can be extracted from the width of the $\rho^{0} \rightarrow \pi^{+} \pi^{-} \pi^{+} \pi^{-}$decay if one assumes that this decay goes over an intermediate $\rho^{0} \sigma$ state.

The two contributions from Compton scattering $\left(\Sigma^{\text {Compt }}\right)$ and $\sigma$ exchange $\left(\Sigma^{\sigma-\text { exch }}\right)$ are shown in Fig. 1, For the estimate shown in Fig. 11 the corresponding $N N \rho$ tensor coupling and $N N \sigma$ coupling strength were taken from the Bonn one-boson-exchange model [66] for nucleon-nucleon scattering $\left[f_{N N \rho}=19.8\right.$ (tensor coupling) and $\left.g_{N N \sigma}=10\right]$. The error band for $\Sigma^{\sigma-\text { exch }}$ is due to 
the relatively large uncertainty in the four- $\pi$ decay of the $\rho$ meson

$$
\operatorname{Br}\left(\rho^{0} \rightarrow \pi^{+} \pi^{-} \pi^{+} \pi^{-}\right)=(1.8 \pm 0.9) \times 10^{-5} .
$$

Nevertheless, from Fig. 1, one sees that the contributions from Compton scattering and $\sigma$-exchange are of different sign and comparable magnitude. For the mean value of the $\operatorname{Br}\left(\rho^{0} \rightarrow \pi^{+} \pi^{-} \pi^{+} \pi^{-}\right)$ branching, they almost cancel completely, and changes of the $\rho$-meson spectral function shown below are insignificant.

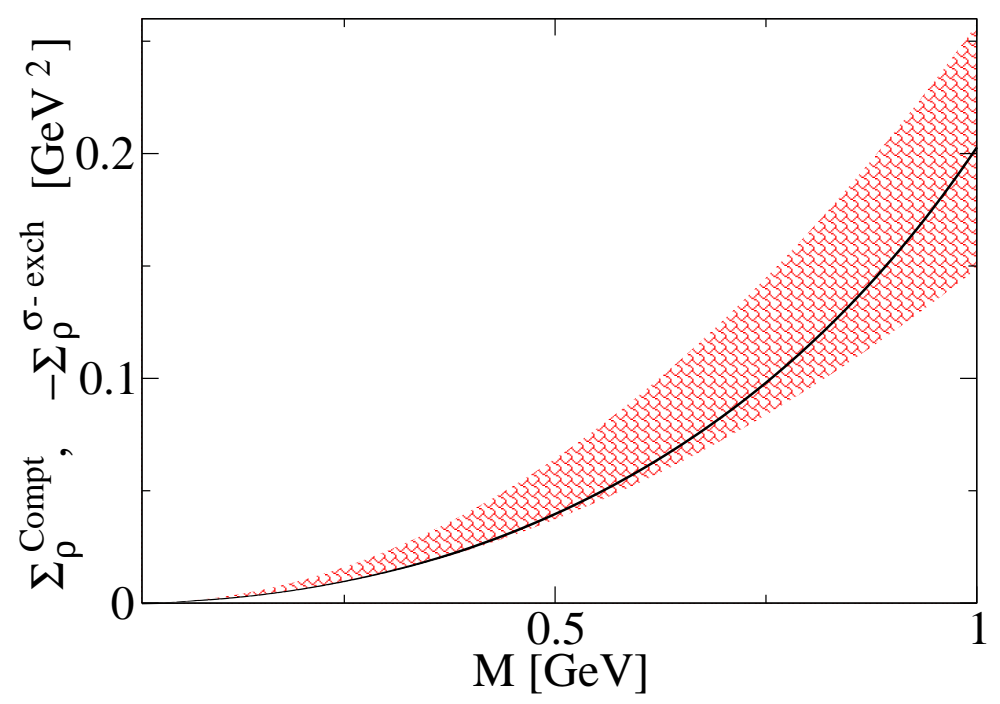

FIG. 1: (Color online) Nonresonant contributions to $\rho$-meson self-energy from Compton scattering amplitude (solid line) and from the amplitude due to exchange by $\sigma$ meson (shaded region). The shaded region corresponds to the error in the branching ratio $\operatorname{Br}\left(\rho^{0} \rightarrow \pi^{+} \pi^{-} \pi^{+} \pi^{-}\right)=(1.8 \pm 0.9) \times 10^{-5}$.

To account for nonresonant contributions to the $\omega$ spectral function within the present scheme, we assume an $\omega \omega \sigma$ coupling three times larger than that for $\rho \rho \sigma$ which is motivated by the comparison with the two-pion coupling. The $N N \omega$ vector coupling $\left(g_{N N \omega}=15.9\right)$ is again taken from the Bonn potential [66]. As can be seen in Fig. 4, the influence of the nonresonant contributions is now more pronounced than in the case of the $\rho$ meson; however, the qualitative features of the spectral distributions are not changed.

The Breit-Wigner amplitudes decrease as $1 / s$ with increasing $s$. Such a parametrization ensures the change of the resonance phases by $\pi$ from low to high energies. The $\sigma$-meson exchange generates the scalar mean field, which is known to be important in the modification of the nucleon masses [1]. It plays an important role in our scheme too. The component of the amplitude connected to the $\sigma$-meson exchange remains constant for $s \rightarrow \infty$.

\section{C. $\rho$-meson spectral function}

In the following, we discuss first the $\rho$ meson. Figure 2 shows the $\rho$ spectral function in nuclear matter at nuclear saturation density $\rho_{0}=0.16 \mathrm{fm}^{-3}$. Longitudinal $\left(\mathcal{A}^{L}\right)$ and transverse $\left(\mathcal{A}^{T}\right)$ spectral functions are found to be rather similar. This means that unpolarized spectral functions can be used in the calculations of dilepton spectra. 


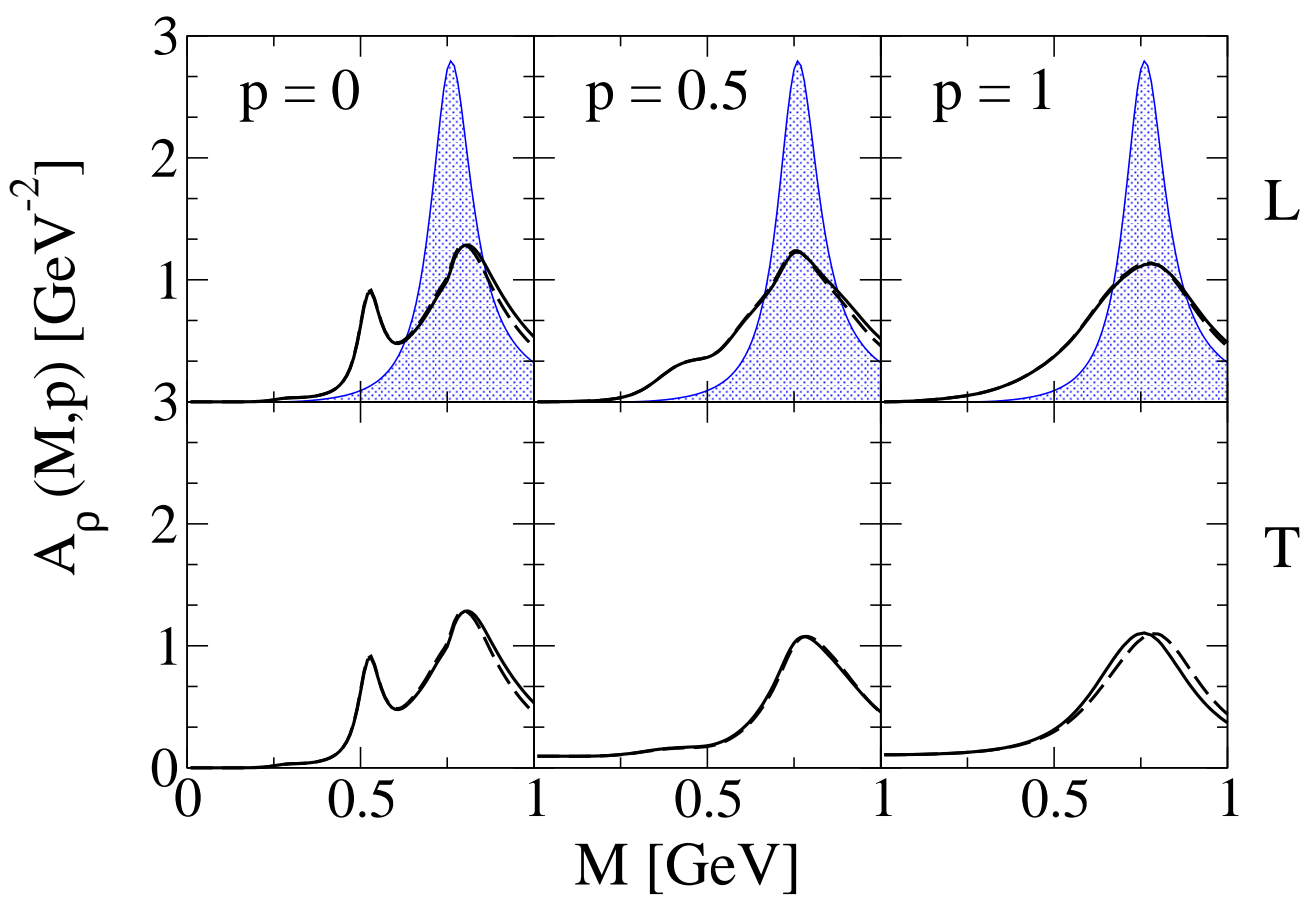

FIG. 2: (Color online) Longitudinal $(L)$ and transverse $(T) \rho$ spectral functions in nuclear matter at saturation density for various momenta $p$ (in $\mathrm{GeV}$ ). Dashed lines stand for the resonance approximation, solid lines represent calculations that also included the nonresonant contributions. The shaded area shows the vacuum spectral function.

We observe a slight upward mass shift of the $\rho$ and a substantial broadening. At low momenta, the spectral functions show a clear two-peak structure which vanishes with increasing vector meson momentum. The results shown in Fig. 2 are in qualitative and even quantitative agreement with previous calculations based on the resonance model assumption [21]. Although the various approaches are based on different ways to describe the corresponding transition form factors, eVMD in the present case, and parameters are partially fixed in different way, this fact demonstrates the stability of the essential features predicted by these types of models.

The emerging two-peak structure can be understood as follows. The value and sign of the selfenergy $\Re \Sigma_{V}$ depend on the pole positions of the particular resonances. If the vector meson mass squared is small, the invariant mass of vector meson plus nucleon is below the pole masses of the relevant nucleon resonances. Therefore the real part of the vector meson self-energy is negative. This is a typical example for level repulsion (vector meson plus nucleon and nucleon resonance). Consequently, the factor $\left(m^{2}-m_{V}^{2}-\Re \Sigma_{V}\right)^{2}$ in the denominator of the vector meson spectral function, Eq. (11), is small or even equal to zero. Thus the first peak in the spectral function emerges at a vector meson mass around $0.5 \mathrm{GeV}$. The major contribution, which generates the first peak, comes from the $N^{*}(1520)$, which is in agreement with the findings reported in Ref. [21].

If the vector meson mass squared lies in the vicinity of its vacuum value $m_{V}^{2}$, the invariant mass of vector meson plus nucleon lies above the pole masses of the relevant nucleon resonances and the real part of the vector meson self-energy is positive. Thus we obtain the second peak in the spectral function at a vector meson mass slightly above $m_{V}$.

At high vector meson momenta, the invariant mass of the vector meson plus nucleon is always 
above the pole masses of the relevant nucleon resonances. As a result, the spectral function has only one single peak slightly above $m_{V}$.

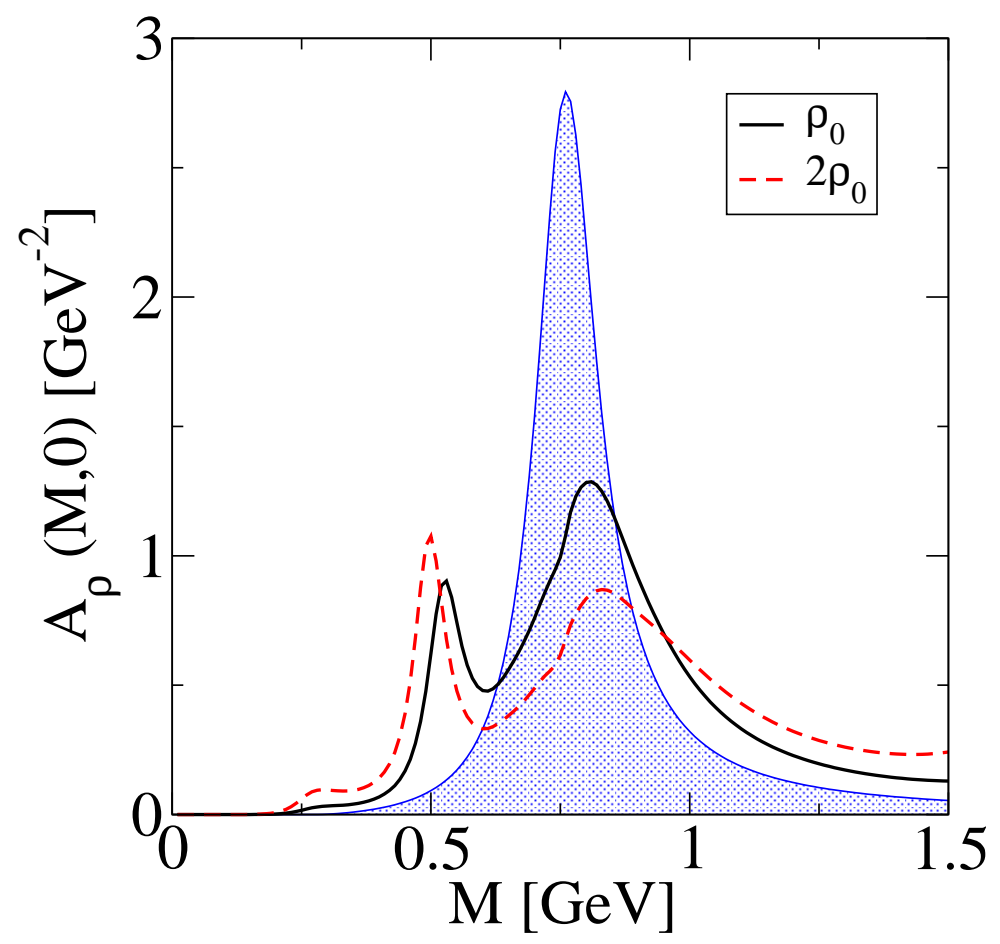

FIG. 3: (Color online) Unpolarized $\rho$ meson spectral function at rest in nuclear matter at saturation density and twice saturation density. The shaded area displays the vacuum spectral function.

Figure 3 displays finally the dependence of the $\rho$-meson spectral function on nuclear density. It shows the unpolarized $\rho$-meson spectral function at rest at $\rho_{0}$ and at $2 \rho_{0}$ nuclear density. With increasing density, we observe a further shift of strength away from the original pole mass; i.e., the first branch in spectral distribution is slightly enhanced and even shifted to lower masses, while the second peak is slightly shifted upward at $2 \rho_{0}$ compared to $\rho_{0}$ and also additionally broadened.

In this context, it should be noted that the resonance model predictions stand in contrast to the EFT coupled-channel calculations of Ref. [19], which predict no significant medium dependence of the $\rho$, concerning neither a mass shift nor a broadening. The reason that in the approach of Ref. [19] much less strength is shifted to lower masses lies mainly in the much weaker coupling to the $N^{*}$ (1520) found in Ref. [19]. For this resonance, the value of $\Gamma_{N \rho} \sim 2 \mathrm{MeV}$ [19] has to be compared with $\Gamma_{N \rho} \sim 25 \mathrm{MeV}$ from Refs. [21, 46]. The latter value, however, agrees with that of the PDG [67] and the Manley and Saleski analysis [62].

\section{D. $\omega$-meson spectral function}

For the $\omega$ meson, we observe a behavior that is principally similar to that of the $\rho$ meson (see Fig. (4). Transverse and longitudinal spectral functions are similar. In both cases, the $\omega$ pole mass is slightly shifted upward, and the $\omega$ is substantially broadened around its quasiparticle pole. At $\rho_{0}$ we obtain an in-medium $\omega$ width of $300 \mathrm{MeV}$. 
As in the case of the $\rho$, the coupling to low lying resonances leads to the appearance of a first peak in the spectral function which lies around 0.5-0.55 GeV. With increasing momentum, this peak is washed out and disappears finally. However, in the case of the $\omega$, the influence of nonresonant contributions is found to be much stronger than for the $\rho$. The nonresonant contributions tend to increase the repulsive mass shift of the $\omega$ pole, and they strongly suppress the first peak in the spectral function.

This first branch in the spectral distribution is mainly generated by the $N^{*}(1535)$ resonance. As discussed in detail in Refs. [46, 51], within the NRD+eVMD model a strong $N^{*}(1535) N \omega$ coupling is implied by the available electroproduction and photoproduction data. However, the $N \omega$ decay of this resonance has not been measured directly, and therefore input from quark model predictions had to be used to fix the entire set of eVMD model parameters. Nevertheless, within such a procedure, a strong $N^{*}(1535) N \omega$ coupling seems practically unavoidable. In $p p \rightarrow p p \omega$ production, the large $N^{*}(1535) N \omega$ decay mode leads to substantial contributions in a kinematic regime where the $\omega$ is far off-shell, i.e., at small invariant masses. This is reflected in an enhancement in the cross section around threshold [51]. Existing data [55, 68, 69], however, do not rule out such a behavior. A closer inspection of the experimentally observed background contributions may provide important experimental information concerning this question.

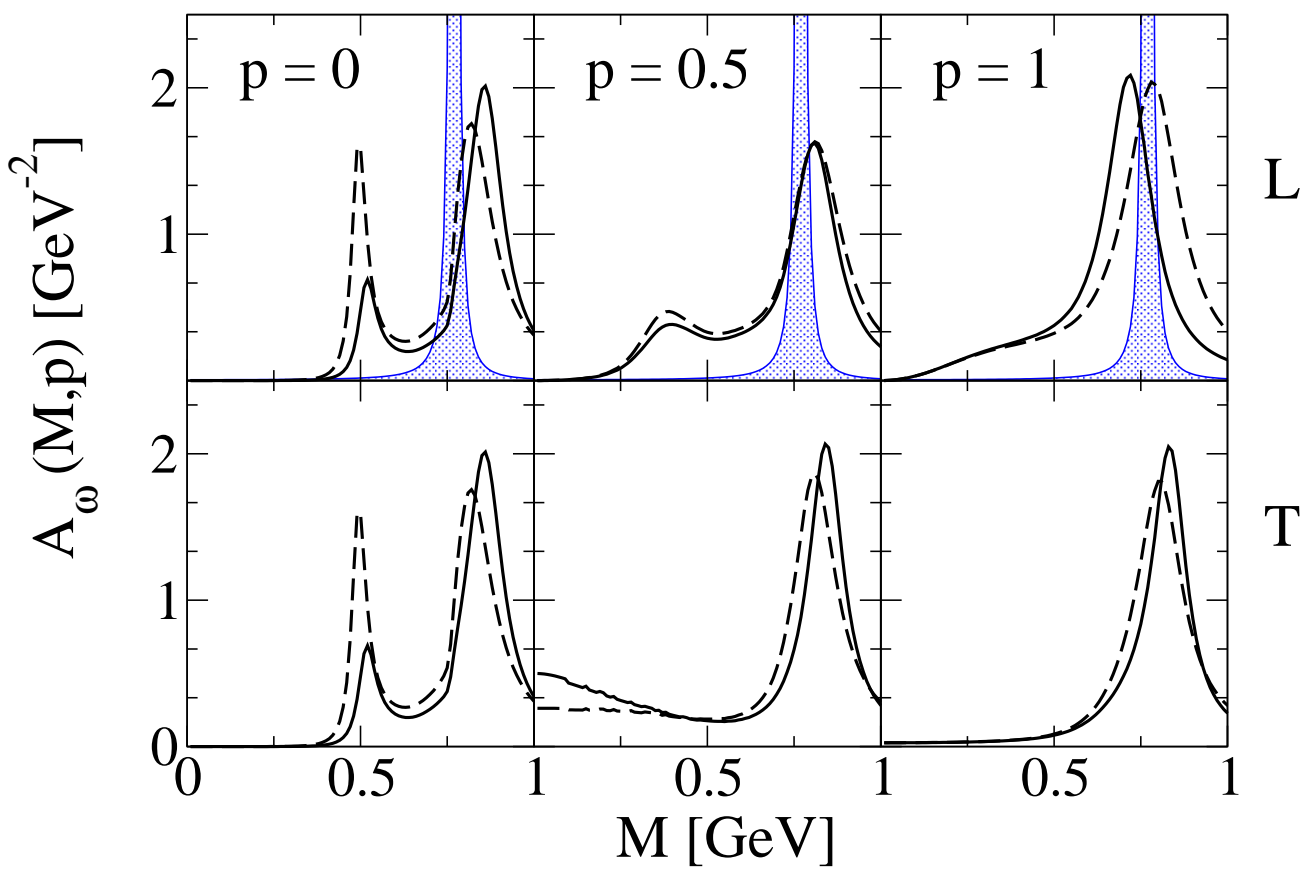

FIG. 4: (Color online) Same as Fig. 2, but for $\omega$ spectral functions.

The nuclear matter density dependence of the $\omega$-meson spectral function is shown in Fig. 5 , Again, the figure shows the unpolarized spectral function at rest at $\rho_{0}$ and at $2 \rho_{0}$ nuclear density. As for the $\rho$ meson, we observe a shift of the second peak which belongs to the original $\omega$ pole toward higher masses with an increase in density, while the first peak is slightly shifted to lower masses. Moreover, the height of the second peak is suppressed by about a factor of 2 .

Comparing this with other works, we should mentioned that in the pure resonance model approach of Ref. [20], no such additional peak was observed. The $\omega$-meson spectral functions 


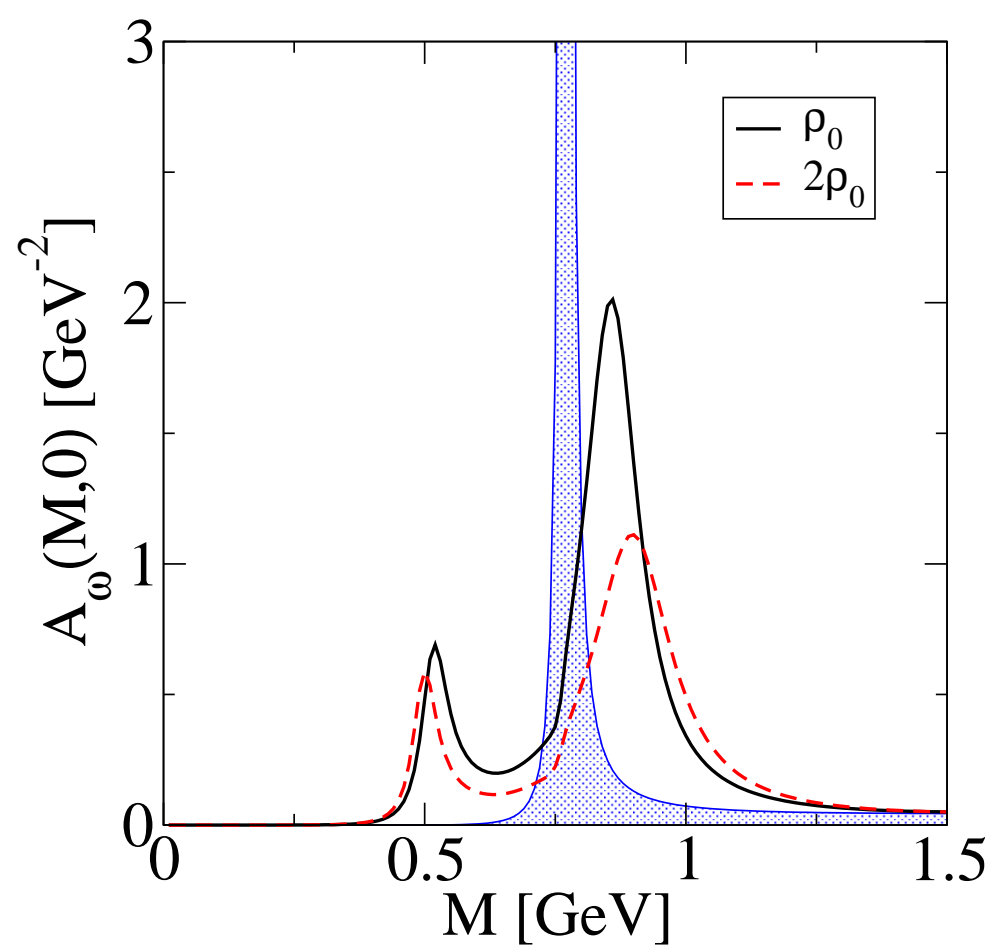

FIG. 5: (Color online) Same as Fig. 3, but for the unpolarized $\omega$-meson spectral function.

obtained within the coupled-channel approach of Ref. [19] and within the coupled-channel $K$ matrix of Ref. [23] have qualitative similarity with those from the present approach. All approaches come practically to the same conclusions: an upward mass shift, a broadening of the $\omega$, and the appearance of an additional branch in the $\omega$ spectral function. This branch appears at the same position and is in both cases generated by the $N^{*}(1535)$. However, in all approaches, the $\omega$ survives as a quasiparticle, at least at moderate densities up to $\rho_{0}$; i.e., there the spectral function is still dominated by the main branch corresponding to the original $\omega$ pole. The predictions for the density dependence of the spectral function are similar on a qualitative level; i.e., when going from one to two times nuclear density, the suppression of the branch corresponding to the $\omega$ pole is of similar size.

However, on a quantitative level, the models come to different conclusions. While the broadening of the $\omega$ is similar in Refs. [19] and [23], the mass shift is much larger in Ref. [19] $\left(\Delta m_{\omega} \sim 46 \mathrm{MeV}\right.$ at $\left.\rho_{0}\right)$ than in Ref. [23] $\left(\Delta m_{\omega} \sim 10 \mathrm{MeV}\right.$ at $\left.\rho_{0}\right)$. In the present case, the in-medium modifications of the $\omega$ meson are even more pronounced than in Refs. [19, 23]; i.e., the broadening and the upward mass shifts are larger $\left(\Delta m_{\omega} \sim 75 \mathrm{MeV}\right.$ at $\left.\rho_{0}\right)$.

A comparison with predictions from QCD sum rules [8, 9] turns out to be difficult because the $\omega$ properties depend strongly on higher order condensates. Sum rules leave space for upward and downward mass shifts, and the parameters related to the higher order terms in the operator product expansion have to finally be fixed from experiments [9]. Moreover, these approaches assume that the $\omega$ maintains its quasiparticle properties. However, due to the distinct two-peak structure of the present spectral distributions, it is not possible to assign a common mass shift to an $\omega$ quasiparticle pole. 


\section{E. In-medium resonances: Role of self-consistency}

As the next step, we took into account the changes induced by the in-medium vector mesons on the total width of the nucleon resonances. This leads to a self-consistent determination of the self-energies of the vector mesons in nuclear matter.

The results shown in the previous section correspond to the first iteration, if considered in the context of a self-consistent calculation. In the second iteration, the in-medium widths of the nucleon resonances $\Gamma_{R}^{*}$ are determined by insertion of the in-medium spectral functions of the vector mesons resulting from the first iteration. Because the latter depend on the momentum of vector meson with respect to nuclear medium $\mathbf{p}$, the in-medium widths of the nucleon resonances $\Gamma_{R}^{*}$ will depend on the resonance momentum $\left|\mathbf{p}_{R}\right|$, that is,

$$
\Gamma_{R}^{*}\left(s,\left|\mathbf{p}_{R}\right|\right)=\Gamma_{R}^{\mathrm{tot}}(s)+\sum_{V} \int \Gamma_{R N V}(s, M) \Delta \mathcal{A}_{V}(M,|\mathbf{p}|) d M^{2} \frac{d \Omega}{4 \pi},
$$

where $\Delta \mathcal{A}_{V}$ refers to the modification of vector meson spectral functions with respect to the vacuum ones. $|\mathbf{p}|$, among other things, depends on $\left|\mathbf{p}_{R}\right|$ and on the orientation of the decay products momenta with respect to the direction of the resonance momentum.

Doing so, nucleon-resonance scattering terms leading to the broadening of the resonances are produced [21]. The vector meson self-energies are then calculated from Eqs. (11)-(3) using $\Gamma_{R}^{*}\left(s,\left|\mathbf{p}_{R}\right|\right)$ instead of $\Gamma_{R}^{\text {tot }}(s)$. The procedure is repeated until convergence. We find that the convergence is obtained after the third iteration.

As a side result of our self-consistent calculation, we find that the widths of the nucleon resonances are enhanced in medium because the vector meson spectral functions show a significative spectral strength at small invariant masses. A similar outcome emerged from the analysis performed in Ref. [70].

The resulting unpolarized vector meson spectral functions are shown in Figs. 6 and 7 for the $\rho$ and $\omega$ mesons, respectively. They refer to saturation density. We observe that the self-consistent calculation leads predominantly to a reduction of the lower mass peak. This result qualitatively agrees with the findings of Ref. [21], which investigated the role of a self-consistent iteration scheme on the $\rho$-meson spectral function.

\section{F. Experimental situation}

Experimental constraints on the in-medium $\omega$ spectral function can presently be derived from the CB-TAPS $(\gamma+A)$ experiments [40], the $p+A$ measurements at KEK [41, 42], and the heavy-ion dilepton experiments. The dilepton measurements of the DLS Collaboration in $\mathrm{C}+\mathrm{C}$ and $\mathrm{Ca}+\mathrm{Ca}$ at $1 A \mathrm{GeV}$ [32] suffer from too low mass resolution in the vicinity of the $\omega$ peak in order to make precise statements on the $\omega$ in-medium width. However, there is no doubt that the explanation of the DLS data requires a substantial broadening of the $\omega$ spectral function. The analysis of Ref. [36] showed that the DLS data are compatible with a rather large $\omega$ width; i.e., $\Gamma_{\omega}^{\text {tot }} \sim 150-300$ $\mathrm{MeV}$. The first data from HADES [38] will be analyzed in the next section.

As discussed in Ref. [43], the interpretation of the $p+\mathrm{C}$ and $p+\mathrm{Cu}$ KEK dilepton data [41, 42] suffers from the high initial proton kinetic energy of $12 \mathrm{GeV}$. This means that vector mesons are produced with high momenta $(p>1 \mathrm{GeV})$ and in particular the $\omega$ decays at low nuclear densities 


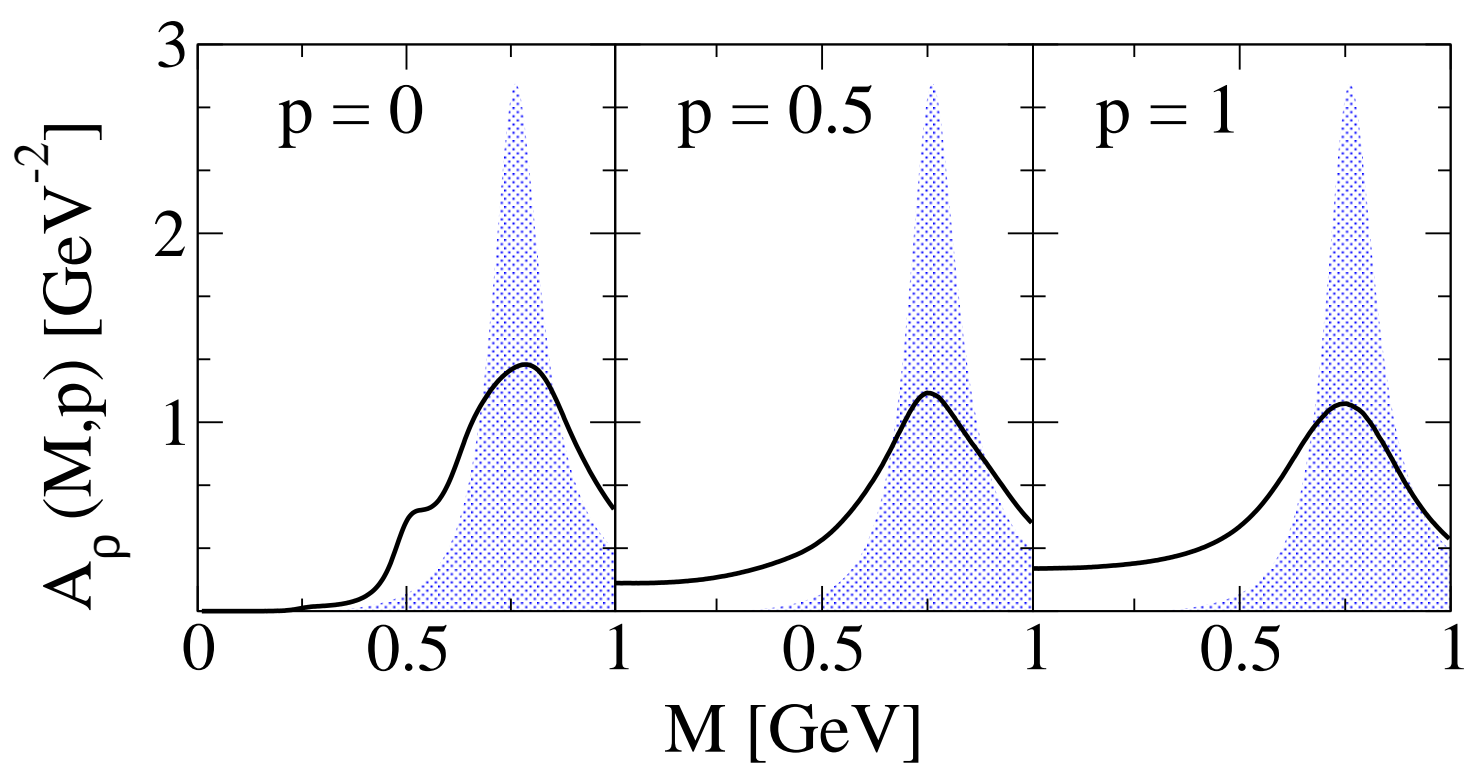

FIG. 6: (Color online) Unpolarized spectral functions of the $\rho$ meson in nuclear matter at saturation density for various momenta $p$ (in $\mathrm{GeV}$ ). The broadening of the nucleon resonance widths induced by the in-medium spectral properties of the vector mesons is taken into account and a self-consistent calculation is performed. The shaded area shows the vacuum spectral function.

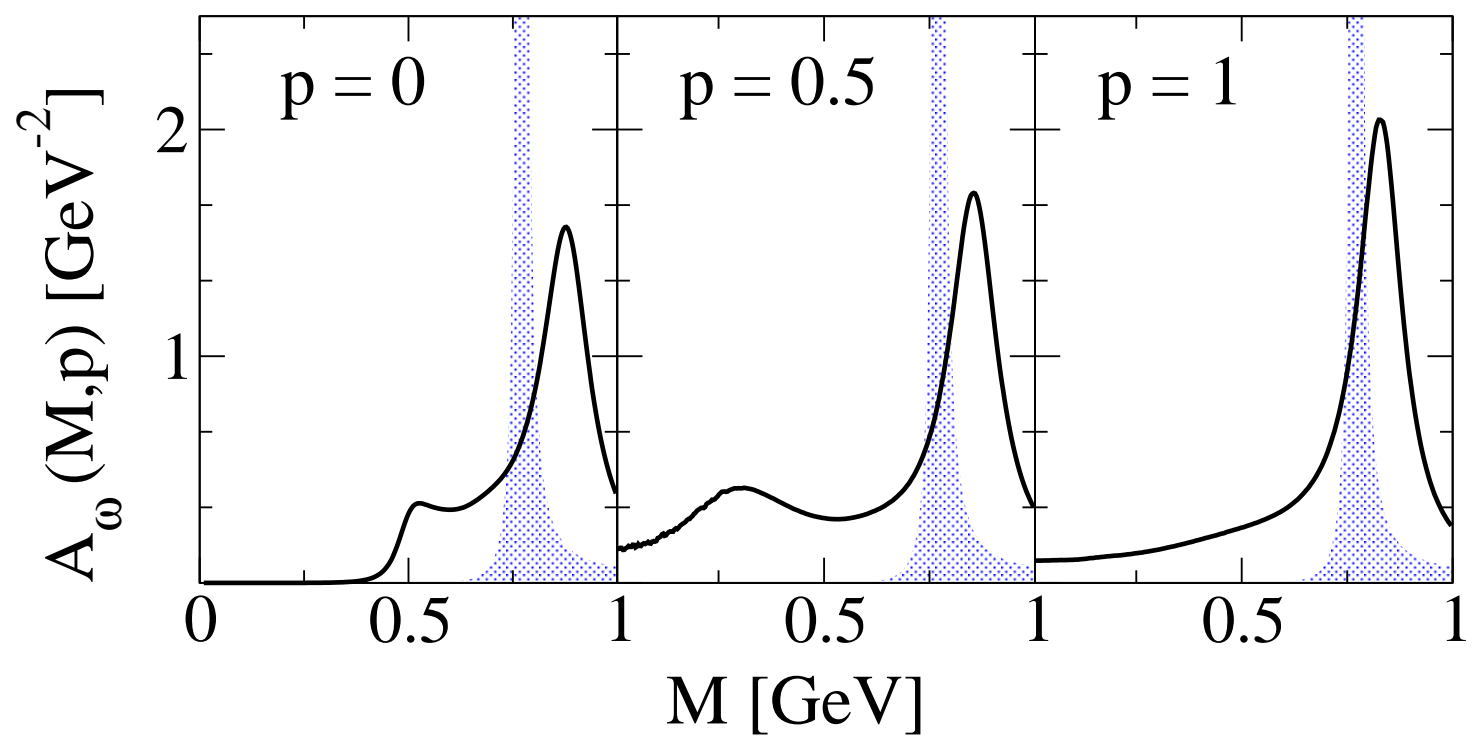

FIG. 7: (Color online) Same as Fig. 6, but for the $\omega$ meson.

or even outside the target nucleus. Nevertheless, the KEK study observed a substantial difference of the dilepton spectrum with respect to the standard sources below the $\rho / \omega$ peak. On a qualitative level, these data support a picture as predicted by the resonance model, i.e., a substantial shift of spectral strength to smaller masses.

However, the resonance model and coupled-channel predictions for the $\omega$ spectral function contradict, at least partially, the results of a recent photoproduction experiment carried out by the CBELSA/TAPS Collaboration [40]. The experiment indicates a downward mass shift of about 
$\Delta m_{\omega} \sim-80 \mathrm{MeV}$ at $\rho_{0}$, whereas the coupled-channel [19, 23] and resonance model calculations predict a more or less pronounced repulsive shift ranging from +10 to $+80 \mathrm{MeV}$. The collisional broadening extracted by CBELSA/TAPS is moderate; i.e., $\Delta \Gamma_{\omega} \sim 50 \mathrm{MeV}$. Since this experiment has been carried out at beam energies of $0.64-2.53 \mathrm{GeV}$, a similar argument as for the KEK experiment applies, at least for the high energies, namely, that one has to carefully account for the energy dependence of the $\omega$ spectral function and to follow the paths of the $\omega$ decays.

Recent $\gamma+A$ measurements from the CLAS Collaboration [71] at the Thomas Jefferson National Accelerator Facility, carried out at photon energies $E_{\gamma}=0.6-3.8 \mathrm{GeV}$ on light (C) and heavier (Fe) targets, find no signatures for a vector meson mass shift for the $\rho$ and $\omega$ mesons but indicate a collisional broadening of the $\rho$ by $50-70 \mathrm{MeV}$.

In terms of a simple BR scaling interpretation $m_{V}^{*}=m_{V}\left(1-\alpha \rho_{B} / \rho_{0}\right)$ which should hold in common for both the $\rho$ and the $\omega$ meson, the best fits to the various experiments yield at present a divergent picture: $|\alpha| \sim 0.13$ (CBELSA/TAPS [40]), $|\alpha| \sim 0.092 \pm 0.002$ (KEK-PS E325 [42]), and $|\alpha| \sim 0.02 \pm 0.02$ (CLAS [71]).

This apparent contradiction implies already that the mass shift scenario à la Brown-Rho is too simple, and, consistent with the NA60 heavy-ion data [30], the vector mesons develop more complex spectral properties.

\section{G. Realization within the transport approach}

A first attempt to introduce in-medium spectral functions of the vector mesons in a transport description for intermediate energy heavy-ion collisions was performed in Ref. [34]. The in-medium dilepton rate was thereby expressed in terms of the $\rho$ meson in-medium spectral function. The proportionality was achieved by including the medium effects at the level of production channels. However, to avoid double counting, this required switching off the explicit $\rho$ meson production channels included in the self-energy calculations, which have been implicitly accounted for in terms of the in-medium spectral function. In particular, the decays of the $\rho$ mesons produced in baryonbaryon collisions and meson-baryon interactions were not included explicitly.

In the language of a resonance model, this would mean, e.g., that since $V+N \rightarrow R$ is a (dominant!) contribution to the self-energy, the $R \rightarrow N V \rightarrow N e^{+} e^{-}$decay should not be included explicitly, if one would operate as in Ref. 34]. However, nucleon resonances are important dynamical degrees of freedom of a transport approach. In particular, in heavy-ion collisions at intermediate energies, the medium is dominated by nucleons and nucleon resonances, and to neglect an explicit (dynamical) treatment of the latter in the determination of vector meson production to obtain a direct proportionality to the vector meson spectral function is a questionable procedure. Moreover, it was already pointed out in Ref [18] that such a treatment [34] might not be realistic.

Here we adopt an alternative approach to extract information on the in-medium vector meson spectral functions from dilepton emission in heavy-ion collisions. The idea is to restrict propagation and mutual interactions to the dynamical degrees of freedom within the transport approach. Vector mesons and their interactions are treated as perturbative degrees of freedom within the transport description. This gives us the possibility of including the corresponding vector meson in-medium modifications on a microscopic level. The philosophy behind this approach is similar to that of the approach pursued in Ref. [18, 31], which derived local dilepton emission rates from the decay rates of the corresponding sources within the framework of an expanding fireball model. 
To be more precise, within the NRD model the vector meson production channels are nucleon resonances which are treated as explicit, i.e., as dynamical degrees of freedom in the transport code up their decay. Dilepton emission takes place via resonance Dalitz decays (eVMD) where the vector mesons enter as virtual particles the $R N \gamma^{*}$ vertices. In the medium, the vector mesons entering into the vertex form factors are modified by the vector meson self-energy, determined for the conditions, i.e., at the density where the resonance decay takes place. Thus the presence of the medium changes the branching ratios for the nucleon resonance Dalitz decays. This modification, however, is not directly proportional to the vector meson spectral function but rather to the inmedium form factors. In this approach, e.g., vector meson absorption processes are taken into account microscopically, although not dynamically, in terms of the imaginary part of the vector meson self-energy, which determines the corresponding collisional broadening.

By this procedure, one avoids the complicated and yet not fully resolved problem of a consistent off-shell propagation of the vector mesons within semiclassical transport models [34, 72]. A drawback is certainly that one loses in this picture information on the dynamical propagation of the vector mesons. Consequently, their in-medium properties are determined by the conditions at the decay points of the nucleon resonances. Since the vector mesons appear only as intermediate states in the resonance decay rates, it is, on the other hand, much easier to include spectral functions and to keep quantum effects which are lost in semiclassical approaches, even when off-shell effects are taken into account $[73,74]$.

The in-medium spectral properties discussed in the next subsection are determined in a local density approximation (LDA). In RQMD, the local baryon density $\rho_{B}$ is determined by the summation over Gaussian wave packets of all nucleons and resonances (it should not be mixed up with the interaction density used in RQMD for the determination of the intranuclear forces [75]). Thus the determination of the baryon density does not require local equilibrium as in hydrodynamics, but dependences on particular nonequilibrium effects such as phase-space anisotropies [76] or memory effects [77] are neglected. As usually done at intermediate energies, an explicit temperature dependence of spectral properties is neglected as well. It is, however, possible to extract local temperatures from transport simulations, either by fitting hot Fermi distributions to local momentum space configurations [78] or by performing thermal model fits to local hadron abundances and spectra [79]. Although both procedures are connected with an extremely high numerical effort, it is thus possible to include density- and temperature-dependent spectral functions into transport simulations. Since temperature effects have been found to dominate at SPS energies [31], such an extension will be necessary for an application of the present model to, e.g., NA60 data.

\section{H. In-medium dilepton emission rates}

Because of the $P$ invariance of the electromagnetic interaction, resonances with arbitrary spin have only three independent helicity amplitudes in the $\gamma^{*} N \rightarrow R$ transitions. This means that there are three independent scalar functions to fix the vertices. The three scalar functions arising from the decomposition of the $\gamma^{*} N \rightarrow R$ vertex over the Lorentz vectors and the Dirac matrices are functions of the mass squared $M^{2}$ of the virtual photon and are called covariant form factors. In the eVMD model, each of these covariant form factors is expressed in a gauge invariant way (see Appendix) as a linear superposition of the contributions from the intermediate vector mesons of the $\rho$ and $\omega$ family. In contrast to the naive VMD, in which only the $\rho$ and $\omega$ ground states are 
taken into account, eVMD includes radial excitations $\rho(1450), \rho(1700)$, etc., which interfere with the ground-state $\rho$ mesons in radiative processes. The corresponding transition form factors are given by [46]

$$
F_{k}^{( \pm)}\left(M^{2}\right)=\sum_{i} \mathcal{M}_{k i}^{( \pm)}
$$

where $k=1, \ldots, 3$ stands for each of the form factors, $( \pm)$ denotes states of normal and abnormal parity, respectively, and the sum is over the intermediate mesons. The $\Delta$ resonance form factors have contributions from only the $\rho$-meson family, whereas the nucleon resonances receive contributions from the $\rho$ and $\omega$ mesons. For a resonance of spin $J=l+1 / 2$, the total number of vector mesons is $l+3$. The amplitude

$$
\mathcal{M}_{k, i}^{( \pm)}=h_{k i}^{( \pm)} \frac{m_{i}^{2}}{m_{i}^{2}-i m_{i} \Gamma_{i}-M^{2}}
$$

represents the contribution of the $i$ th vector meson to the form factor of type $k$. The residues $h_{k i}^{( \pm)}$contain the free parameters of the model. They are constrained by the requirement that the asymptotic expression of the form factors is consistent with the quark counting rules [80]. For each form factor, the quark counting rules reduce the number of free parameters from $l+3$ to 2 for $k=1$ and to 1 for $k=2,3$. The remaining parameters are fixed by fitting the available photoproduction and electroproduction data and using results of the multichannel partial-wave analysis of the $\pi N$ scattering. Where experimental data are not available, predictions of the nonrelativistic quark models are used as an input.

The $\Gamma\left(R \rightarrow N \gamma^{*}\right)$ decay width can be written in terms of three transition form factors (magnetic, electric, and Coulomb) for a resonance with spin $J>1 / 2$ and two for $J=1 / 2$. The matrix elements connecting the former with the covariant form factors are explicitly listed in Ref. [46].

In this representation, the insertion of the in-medium properties of the $\omega$ and $\rho$ vector mesons is straightforward. In the medium, the transition amplitudes $\mathcal{M}_{k, i}^{( \pm)}(i=\rho, \omega, \ldots)$ are directly modified by the in-medium self-energy and read

$$
\mathcal{M}_{k, i=V}^{( \pm)}=h_{k V}^{( \pm)} \frac{m_{V}^{2}+\Re \Sigma_{V}^{\text {tot }}}{m_{V}^{2}+\Re \Sigma_{V}^{\text {tot }}+i \Im \Sigma_{V}^{\text {tot }}-M^{2}}
$$

We include the self-energy contributions for the ground-state $\rho$ and $\omega$ mesons in the transition. For the excited states $\rho^{\prime}, \rho^{\prime \prime}, \ldots$, the self-energies are unknown, and thus we keep for these states their vacuum properties.

As in Ref. [58], we also consider scenarios in which the self-energy is based on different model assumptions, namely, a simple Brown-Rho (BR) or Hatsuda-Lee scaling of the vector meson masses [5, 6] and a collisional broadening of the vector meson widths. In the latter case, the self-energies are given by

$$
\begin{aligned}
& \Im \Sigma_{V}^{\text {tot }}=-m_{V}\left(\Gamma_{V}^{(0)}(M)+\Gamma_{V}^{\text {coll }}\left(\rho_{B}, M\right)\right), \\
& \Re \Sigma_{V}^{\text {tot }}=0 .
\end{aligned}
$$

In this context, we want to stress that in Eqs. (16), the energy dependence due to the twoor three-pion decay of the vector meson is kept in the vacuum contribution to the total width, 
while the collisional broadening due to the interaction with the surrounding nucleons is absorbed into a density- and energy-dependent part. The issue of the energy dependence of the collisional width will be discussed in detail in the next section. The BR scaling is introduced through the replacement $m_{V} \rightarrow m_{V}^{*}=m_{V}\left(1-\alpha \frac{\rho_{B}}{\rho_{0}}\right)$, as done, e.g., in Ref. [81]. In particular, in this case, one has

$$
\Re \Sigma_{V}^{\text {tot }}=\left(m_{V}-\alpha \frac{\rho_{B}}{\rho_{0}}\right)^{2}-m_{V}^{2} .
$$

As usual, the mass shift entering into the real part can be adjusted by the parameter $\alpha$. As in the case of full spectral functions, the self-energy components enter into the amplitudes (15). In this context, it is important to note that the modification of the amplitudes (15) leads to a coherent summation of the $\rho$ and $\omega$ spectral functions in the transition form factors (13). Doing so, this approach goes beyond the standard-even off-shell-transport approach where spectral properties are treated at the level of cross sections [34, 72, 73]. The latter always leads to an incoherent summation of the contributions from different hadrons.

The self-energy appearing in Eq. (15) is a function $\Sigma_{V}^{\text {tot }}\left(M,|\mathbf{p}|, \rho_{B}\right)$ of the vector meson running mass, the modulus of its three-momentum in the nuclear matter rest frame, and the local density of the surrounding matter. In the rest frame $L^{*}$ of a resonance $R$ with mass $\mu$, decaying into a nucleon and a vector meson of mass $M$, the modulus $\left|\mathbf{p}^{*}\right|$ of the momentum of the meson is fixed by energy conservation. If $\mathbf{p}_{R}$ is the momentum of the resonance $R$ in the c.m. frame $L$ of the colliding nuclei and $\mathbf{v}_{R}=\mathbf{p}_{R} / \sqrt{\mathbf{p}_{R}^{2}+\mu^{2}}$ is its velocity, the vector meson momentum in $L$ is given by the Lorentz transformation

$$
|\mathbf{p}|^{2}=\left(\gamma_{R}\left|\mathbf{v}_{R}\right| E^{*}+\gamma_{R} p_{L}^{*}\right)^{2}+p_{T}^{* 2}
$$

where

$$
\begin{aligned}
& p_{L}^{*}=\left|\mathbf{p}^{*}\right| \cos \theta, \\
& p_{T}^{*}=\left|\mathbf{p}^{*}\right| \sin \theta,
\end{aligned}
$$

with $\theta$ being the polar angle of the meson in $L^{*}$ if one chooses the $z$ axis of this frame pointing in the direction of $\mathbf{v}_{R}$. Since $\left|\mathbf{p}^{*}\right|$ is fixed, in terms of the $L$ frame variables, one has $\Sigma_{V}^{\text {tot }}=$ $\Sigma_{V}^{\text {tot }}\left(M, \cos \theta, \rho_{B}\right)$, and the decay amplitude averaged over the angles reads

$$
\Gamma\left(R \rightarrow N \gamma^{*}\right)\left(\mu, M, \rho_{B}\right)=\int_{-1}^{+1} \frac{d \cos (\theta)}{2} \Gamma\left(R \rightarrow N \gamma^{*}\right)\left(\mu, M, \cos \theta, \rho_{B}\right) .
$$

Equation (21) is implementable in the framework of the Tübingen RQMD transport code. The RQMD code [36, 56, 57] has been extended to include all nuclear resonances with masses below $2 \mathrm{GeV}$, in total $11 N^{*}$ and $10 \Delta$ resonances. A full list with the corresponding masses and decay widths to various channels can be found in Tables III and IV of Ref. [36]. For each resonance, RQMD provides the values of the three-momentum components (necessary to perform the Lorentz boost), the mass (distributed over a Breit-Wigner), and the local density of the surrounding matter at the decay point.

Since vector mesons play in the eVMD model the role of intermediate virtual particles, their off-shellness is fully taken into account in a consistent manner.

The model can be applied to dilepton production in heavy-ion reactions. In the energy range of a few $A \mathrm{GeV}$, one can identify three main classes of processes that lead to dilepton emission: 
nucleon-nucleon bremsstrahlung, decay of light unflavored mesons, and decay of nucleon and $\Delta$ resonances. Dilepton production through the bremsstrahlung mechanism has been studied in detail in Ref. [82]. For the energy range of interest in this work, bremsstrahlung contributes in a significant way only at small invariant masses to the dilepton spectrum. By far, the dominant contributions result from diagrams that involve the excitation of an intermediate $\Delta$ resonance. Within the present framework, the inclusion of such contributions would, however, lead to a double counting and therefore we omit explicit bremsstrahlung contributions. Recently, the quantitative importance of bremsstrahlung contributions has again been discussed in Ref. [44], however, with results contradictory to those in Ref. [82].

At incident energies of a few $A \mathrm{GeV}$, the cross sections for meson $\mathcal{M}=\eta, \eta^{\prime}, \rho, \omega, \phi$ production are small, and these mesons do not play an important role in the dynamics of heavy-ion collisions. Their production can thus be treated perturbatively, in contrast to the case of the pion. The decay to a dilepton pair takes place through the emission of a virtual photon. The differential branching ratios for the decay of a meson to a final state $X e^{+} e^{-}$can be written

$$
d B(\mu, M)^{\mathcal{M}, \pi \rightarrow e^{+} e^{-} X}=\frac{d \Gamma(\mu, M)^{\mathcal{M}, \pi \rightarrow e^{+} e^{-} X}}{\Gamma_{\text {tot }}^{\mathcal{M}, \pi}(\mu)},
$$

with $\mu$ the meson mass and $M$ the dilepton mass. Three types of such decays have been considered: direct decays $\mathcal{M} \rightarrow e^{+} e^{-}$, Dalitz decays $\mathcal{M} \rightarrow \gamma e^{+} e^{-}$and $\mathcal{M} \rightarrow \pi(\eta) e^{+} e^{-}$, and four-body decays $\mathcal{M} \rightarrow \pi \pi e^{+} e^{-}$. A comprehensive study of the decay of light mesons to a dilepton pair has been performed in Ref. [49]. Assuming a NRD model for the production of $\rho$ and $\omega$ mesons, the remaining decay channels that are most important quantitatively for heavy-ion collisions at $1 A$ and $2 A \mathrm{GeV}$ are $\pi^{0} \rightarrow \gamma e^{+} e^{-}$and $\eta \rightarrow \gamma e^{+} e^{-}$.

In terms of the branching ratios for the Dalitz decays of the baryon resonances, the cross section for $e^{+} e^{-}$production from the initial state $X^{\prime}$ together with the final state $N X$ can be written as

$$
\frac{d \sigma(s, M)^{X^{\prime} \rightarrow N X e^{+} e^{-}}}{d M^{2}}=\sum_{R} \int_{\left(m_{N}+M\right)^{2}}^{\left(\sqrt{s}-m_{X}\right)^{2}} d \mu^{2} \frac{d \sigma(s, \mu)^{X^{\prime} \rightarrow R X}}{d \mu^{2}} \sum_{V} \frac{d B\left(\mu, M, \rho_{B}\right)^{R \rightarrow V N \rightarrow N e^{+} e^{-}}}{d M^{2}} .
$$

Here, $\mu$ is the running mass of the baryon resonance $R$ with the cross section $d \sigma(s, \mu)^{X^{\prime} \rightarrow X R}$, $d B\left(\mu, M, \rho_{B}\right)^{R \rightarrow V N \rightarrow N e^{+} e^{-}}$is the differential branching ratio for the Dalitz decay $R \rightarrow N e^{+} e^{-}$ through the vector meson $V$. Thus Eq. (23) describes baryon- and pion-induced dilepton production; i.e., the initial state can be given by two baryons $X^{\prime}=N N, N R, R^{\prime} R$ or it runs through pion absorption $X^{\prime}=\pi N$. In the resonance model, both processes are treated on the same footing by the decay of intermediate resonances. Medium modifications enter the branching ratio $d B\left(\mu, M, \rho_{B}\right)^{R \rightarrow V N \rightarrow N e^{+} e^{-}}$by affecting the Dalitz decay width $d \Gamma\left(\mu, M, \rho_{B}\right)^{R \rightarrow V N \rightarrow N e^{+} e^{-}} / d M^{2}$. Once the $\Gamma\left(R \rightarrow N \gamma^{*}\right)$ is calculated within the eVMD, the factorization prescription [46] can be used to find the dilepton decay rate

$$
d \Gamma\left(R \rightarrow N e^{+} e^{-}\right)=\Gamma\left(R \rightarrow N \gamma^{*}\right) M \Gamma\left(\gamma^{*} \rightarrow e^{+} e^{-}\right) \frac{d M^{2}}{\pi M^{4}},
$$

where

$$
M \Gamma\left(\gamma^{*} \rightarrow e^{+} e^{-}\right)=\frac{\alpha}{3}\left(M^{2}+2 m_{e}^{2}\right) \sqrt{1-\frac{4 m_{e}^{2}}{M^{2}}}
$$

is the decay width of a virtual photon $\gamma^{*}$ into the dilepton pair with the invariant mass $M$. 
As discussed in Ref. [21], the excitation of particle-hole pairs in the meson spectral function generates resonance-nucleon scattering terms in the resonance self-energy and thus the in-medium broadening of the resonance. We have mentioned that nucleon resonances are dynamically treated in the RQMD model, and resonance-nucleon scattering is explicitly performed. Thus, the inmedium broadening of nucleon resonances is taken into account in the transport approach dynamically. No in-medium spectral functions of the vector mesons are therefore included in the total width $\Gamma_{\text {tot }}^{R}(\mu)$ entering in the branching ratio in Eq. (23).

The real part of the resonance self-energy $\Re \Sigma_{\text {tot }}^{R}$ is included in a phenomenological way through a mean field in which the resonances are propagated. It is therefore assumed that the nucleon resonances feel the same potential as nucleons, which is a standard approximation in present transport models and should be improved in the future. For example, the $R N V$ vertex gives rise to additional Fock contributions which could be included in future work.

An observable tightly connected to a correct treatment of the resonance dynamics in heavy-ion collision transport calculations is provided by the pion multiplicity. For the mass system under consideration, pion multiplicities are reasonably well reproduced by the present description. For example, inclusive $\pi^{+}$cross sections in $\mathrm{C}+\mathrm{C}$ reactions measured by the KaoS Collaboration [83] can be reproduced by the present description within error bars. This gives, at least on a global level, manifest credit to our treatment.

For the $\eta$, we include $\eta$ absorption from the dominating channel $\eta+N \rightarrow N^{*}(1535)$ explicitly. Since chiral perturbation theory predicts practically no modifications of the in-medium $\eta$ mass [84], we do not include a possible $\eta$ mass shift.

To give an impression of the density range relevant for dilepton production in the $\mathrm{C}+\mathrm{C}$ system, Fig. 8 shows the density distribution $d N / d \rho_{B}$ where the nucleon resonance decays into dilepton channels take place. Note that Fig. 8 refers to minimal bias conditions. Triggering on central reactions and/or increasing the system size will help to better explore the high density range. To have a separate look at $\rho$ and $\omega$ production, we distinguish between $N^{*}, \Delta^{*}$, and $\Delta(1232)$ resonances. It can be seen that the highest mass resonances, i.e., $N^{*}$ and $\Delta^{*}$, decay at supranormal densities, while a large fraction of the $\Delta(1232)$ decays take place at lower densities, between $0.5 \rho_{0}$ and $1 \rho_{0}$. In all cases, however, maximal densities up to $3 \rho_{0}-4 \rho_{0}$ are reached. Thus, already the small $\mathrm{C}+\mathrm{C}$ system probes the spectral properties of intermediate vector mesons from $N^{*}$ and $\Delta^{*}$ decays at supranormal densities.

\section{DILEPTON PRODUCTION}

In this section, we provide theoretical calculations of the dilepton emission in heavy-ion collisions at intermediate energy. In particular, we address the reaction $\mathrm{C}+\mathrm{C}$ at $2 \mathrm{~A} \mathrm{GeV}$ for which experimental data have been already released by the HADES Collaboration. The main purpose is to compare calculations that include in-medium effects in a more traditional way, i.e., via Brown-Rho scaling of the vector masses and empirical collisional broadening of the decay width, with results obtained using $\rho$ and $\omega$ mesons described by the in-medium spectral functions of the previous section. New HADES data [85] will be analyzed elsewhere.

In the transport calculation, the reaction has been treated as minimal bias collisions with respective maximal impact parameter $b_{\max }=6.0 \mathrm{fm}$. For the nuclear mean field, a soft momentumdependent Skyrme force $(K=200 \mathrm{MeV})$ is used [75] which provides also a good description of the 


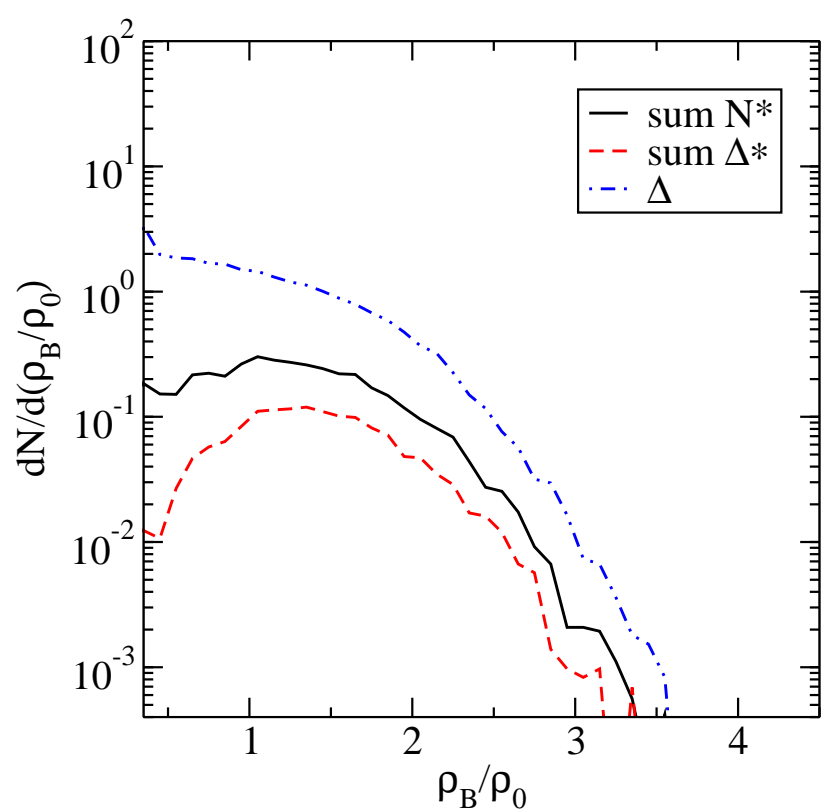

FIG. 8: (Color online) Impact parameter weighted density distribution of the nucleon resonances taken into account in the calculations at their decay point. The distribution of the $N^{*}, \Delta^{*}$, and $\Delta(1232)$ resonances are separately shown.

subthreshold $K^{+}$production in the considered energy range 86]. To perform the comparison with the HADES data, dilepton events originated from the different considered sources were generated in the phase space. After smearing over the experimental momentum resolution, the acceptance filter function provided by the HADES Collaboration was applied. Events with opening angle $\theta_{e^{+} e^{-}} \leq 9^{\circ}$ were rejected, in accordance with the treatment of the experimental data. The spectrum was then normalized to the corresponding $\pi^{0}$ multiplicity.

\section{Vacuum}

We start by addressing the results obtained without any additional medium effects concerning the dilepton production. In Fig. 9, the dilepton spectrum obtained within the vacuum formulation of the NRD+eVMD model is compared with the HADES data [38]. The experimental data are slightly underestimated in the mass region $m_{\pi} \leq M \leq 0.4 \mathrm{GeV}$ and overestimated in the region of the vector meson peak. Indeed, the comparison with DLS data had already shown that the eVMD model in its pure vacuum formulation fails in describing dilepton production in heavy-ion collisions [36]. However, the vacuum calculation is a good reference point for isolating, where possible, those sources that dominantly contribute to the spectrum in a certain invariant mass region. Once the dominant sources have been individuated, it is interesting to look separately at their modifications due to in-medium effects. For this purpose, we also show separately in Fig. 9 the contributions to the spectrum of the decays of the pseudoscalar $\eta$ and $\pi^{0}$ mesons and all the $N^{*}$ as well as the $\Delta$ resonances. In addition, the $\Delta(1232) \rightarrow N e^{+} e^{-}$decay channel is explicitly shown. In what follows, we will investigate the modification of the Dalitz decays of the baryon resonances due to the introduction of the in-medium properties of the $\rho$ and $\omega$ mesons. Since we introduce no in-medium modifications of the $\pi^{0} \rightarrow \gamma e^{+} e^{-}$and $\eta^{0} \rightarrow \gamma e^{+} e^{-}$channels, the contribution to 
the dilepton spectrum from the $\pi^{0}$ and $\eta$ Dalitz decay will remain unchanged in the course of our analysis.

The $\eta$ multiplicity $M_{\eta}(4 \pi)\left[10^{-4}\right]=330$ for the considered reaction $\mathrm{C}+\mathrm{C}$ at $2 A \mathrm{GeV}$ is in agreement with experimental data from TAPS [87]: $M_{\eta}(4 \pi)\left[10^{-4}\right]=294 \pm 46$.

Before coming to the discussion of medium effects, the vacuum results should be examined more closely. As compared to that reported in Ref. [45], we find a higher yield around the $\rho / \omega$ peak region. This enhancement arises in the present model first because of a strong coupling of the $\omega$ to the $N^{*}(1535)$ resonance as discussed in detail in Refs. [36, 58]. Second, additional enhancement results from the implementation of the quark counting rules to the nucleon resonance transition form factors. The quark counting rules are known to be a well-founded consequence of QCD and, furthermore, are required experimentally to match the photon and $\rho$-meson branchings of the nucleon resonances also [47].

A precise estimate of the $\omega$ contribution is particularly important for extracting the $\omega$ meson in-medium width: the underestimation of the dilepton yield gives rise to the underestimation of the width. As we shall see, the in-medium $\omega$ peak is strongly suppressed because of the $\omega$ meson broadening.

The low-mass region is critical for understanding the DLS puzzle. In the present vacuum calculation, we obtain a low-mass dilepton yield that is about a factor of 2 smaller than that in Ref. [45]. Bremsstrahlung cannot explain this deviation, since at $2 A \mathrm{GeV}$ it is marginal whatever maximal cross sections under debate [44] would have been used. At small $M$, the variances in predictions of the present transport models arise from two additional sources, namely, the $\eta$ contribution and the $\Delta$ Dalitz decay: For the $\eta$ decay which dominates the low-mass dilepton yield [36, 45, 58, 88, 89], we obtain quite standard values. The main difference lies therefore in the $\Delta$ Dalitz decay. The present calculation is close to that in Ref. [88] and about a factor of 5 lower than the calculation of Ref. [45]. This point is crucial, since the whole interpretation of the low-mass dilepton spectra depends on this fact.

The problems on the $\Delta(1232)$ Dalitz decay have occurred already at a kinematic level where theoretical calculations of several groups surprisingly disagree with each other pairwise (for a detailed discussion, see Ref. [90]). The dilepton decays can be determined from the radiative decays by factorization, which means that the $N \gamma^{*} \mapsto \Delta$ amplitudes have to be determined first. In this context, it should be noted that from all nucleon resonances, the $N \gamma^{*} \mapsto \Delta(1232)$ transition amplitudes are the best constrained from the experimental point of view (see, e.g., Fig. 20 in Ref. [46]). The $\Delta(1232)$ Dalitz decay is dominated at $M \approx 0$ by the magnetic form factor. The normalization at $M=0$, assuming the dominance of the magnetic transition, is sufficiently precise around $M=0$. With increasing $M$, the Coulomb form factor comes into play. In principle, parametrizations of the $\Delta(1232)$ Dalitz decay should be checked against the available photoproduction data. The quark counting rules constrain the extrapolation to the $M \neq 0$ region.

In the present work, we apply the parametrizations of Ref. [46], which are covariant and kinematically complete, i.e., formulated in terms of magnetic, electric, and Coulomb transition form factors. The $M$ dependence is based on the extended VMD (eVMD) model and constrained by photoproduction and electroproduction data for the form factors, by the transition helicity amplitudes of the nucleon resonances, and when available by the $\rho$ - and $\omega$-meson decay branchings.

The authors of Ref. [45] applied the parametrizations of Ernst et al. [35]. The same parametrization has also been used for the PLUTO event generator of the HADES group [38]. 
However, the work of Ernst et al. [35] considered the magnetic form factor with no $M$ dependence, which is a crude estimation. Furthermore, the kinematic factors of the $\Delta(1232)$ Dalitz decay in Refs. [35, 38, 45] are incorrect, as discussed in Ref. [90].

Therefore, the interpretation of heavy-ion data still suffers from uncertainties unrelated to the complexity of heavy-ion dynamics; i.e., the various parametrizations of the resonance decays used as input in the transport models do not agree with each other.

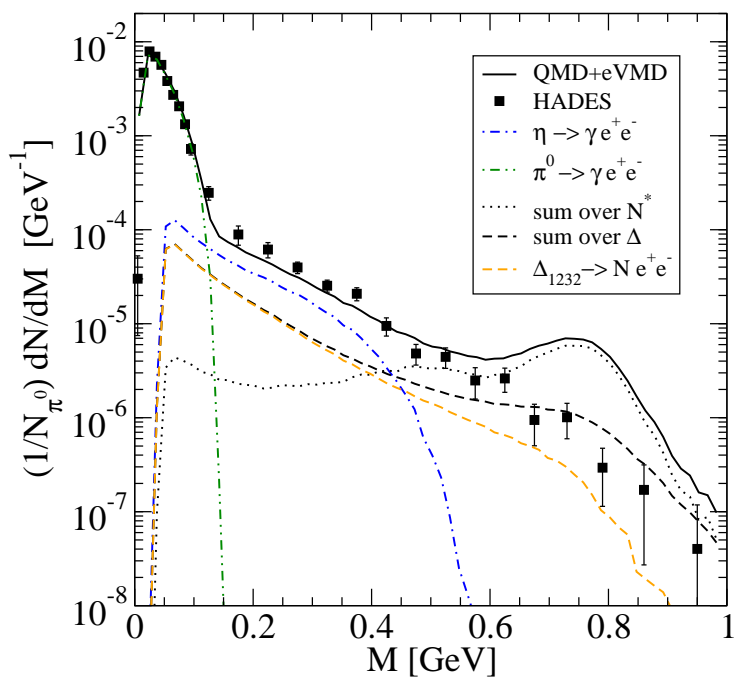

FIG. 9: (Color online) Dilepton spectrum in $\mathrm{C}+\mathrm{C}$ reaction at $2.0 \mathrm{~A} \mathrm{GeV}$ as predicted by the vacuum NRD + eVMD model and compared with HADES data [38]. The contribution of the different types of sources taken into account in the calculation is explicitly shown.

\section{Collisional broadening}

Let us now turn to the introduction of in-medium effects according to the standard treatments and address first Fig. 10, in which the HADES data are compared with calculations where the possible broadening of the vector meson spectral function in medium is effectively taken into account through the introduction of a collisional width $\Gamma_{V}^{\text {coll }}$. We present calculations that use a linear parametrization of the type $\Gamma_{V}^{\mathrm{tot}}\left(\rho_{B}\right)=\Gamma_{V}^{\mathrm{vac}}+\rho_{B} / \rho_{0} \Gamma_{V}^{\mathrm{coll}}\left(\rho_{0}\right)$ to estimate the vector meson in-medium width $\Gamma_{V}^{\text {tot }}\left(\rho_{B}\right)$.

In a first approximation, we make no additional assumption concerning the energy dependence of the in-medium width; i.e., the same energy dependence is assigned to the collisional width as to the vacuum width [36, 58]. In particular, the vector meson thresholds are kept the same as the vacuum ones, namely, $2 m_{\pi}$ and $3 m_{\pi}$ for the $\rho$ and $\omega$ meson, respectively. The approximation will be investigated below. Figure 10(a) refers to the assumption $\Gamma_{\rho}^{\text {tot }}\left(\rho_{0}\right)=200$ and $\Gamma_{\omega}^{\text {tot }}\left(\rho_{0}\right)=60 \mathrm{MeV}$, which reflects the estimates of the CLAS and TAPS experiment for the collisional broadening of the $\rho$ and $\omega$ meson, respectively. Figure 10 (b) refers to the assumption $\Gamma_{\rho}^{\text {tot }}\left(\rho_{0}\right)=250, \Gamma_{\omega}^{\text {tot }}\left(\rho_{0}\right)=125$ $\mathrm{MeV}$. The latter reflects the lower limit estimates emerged from the analysis performed in Ref. [36], where the values of $300 \mathrm{MeV}$ and $200-300 \mathrm{MeV}$, respectly, for the $\rho$ and $\omega$ meson widths at an average density of $1.5 \rho_{0}$ were extracted from fits to the DLS data. 

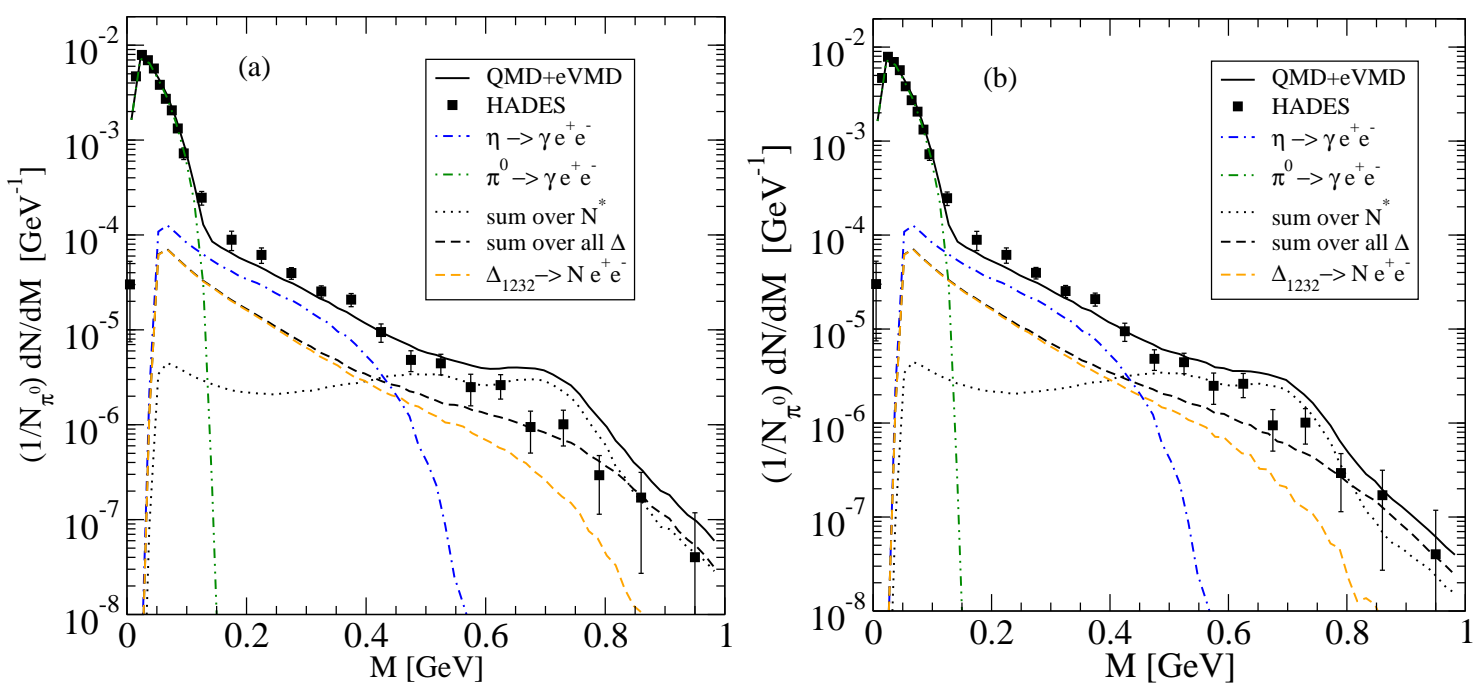

FIG. 10: (Color online) Dilepton spectrum in $\mathrm{C}+\mathrm{C}$ collisions at $2.0 \mathrm{~A} \mathrm{GeV}$ for different values of the inmedium $\rho$ and $\omega$ widths. (a) $\Gamma_{\rho}^{\mathrm{tot}}\left(\rho_{0}\right)=200 \mathrm{MeV}$ and $\Gamma_{\omega}^{\mathrm{tot}}\left(\rho_{0}\right)=60 \mathrm{MeV}$. (b) $\Gamma_{\rho}^{\mathrm{tot}}\left(\rho_{0}\right)=250 \mathrm{MeV}$ and $\Gamma_{\omega}^{\mathrm{tot}}\left(\rho_{0}\right)=125 \mathrm{MeV}$.

We observe a suppression of the peak with respect to the vacuum case, more pronounced in case Fig. 10(b) than in Fig. 10(a). However, in both cases, the experimental data are still overestimated around $M \sim 0.7 \mathrm{GeV}$, mainly due to the still significant contribution of the $N^{*}(1535)$ resonance. The Dalitz decay of this resonance plays a dominant role in the determination of the dilepton spectrum in the region around the vector meson peak, due to its strong coupling to the $\omega$ meson. On the one hand, the HADES data seem to favor a smaller contribution of the $N^{*}(1535)$ resonance in the peak region; on the other hand, however, dilepton production data in $p+p$ collision have been well described under the same assumptions for the coupling to the $N^{*}(1535)$. This shows that the contribution of the $N^{*}(1535)$ Dalitz decay, significant in elementary reactions and thus in vacuum, is partially reduced in heavy-ion collisions thanks to in-medium effects. We conclude that the HADES data suggest a stronger in-medium modification of the $\omega$ properties than the one taken into account in Fig. 10.

Let us now investigate the effect of different choices for the energy dependence of the collisional width. In this context, we would like to point out that the mere fact of having, and facing, a certain freedom in the choice of an energy dependence of the collisional width shows exemplarily the limits that such schematic models carry. Such choices can be based on more or less educated guesses. However, if microscopic calculations of in-medium effects are performed, energy dependences are fixed from theory, which should be fulfilled as a minimal requirement for a consistent investigation of vector meson in-medium properties. Obviously a microscopic calculation of the exact energy dependence of the collisional broadening is equivalent to a full model calculation of the in-medium spectral function. This well be done later on in this work.

For the moment, we investigate the consequences of various approximations on a schematic level. For this purpose, we extract possible energy dependences of the collisional widths on the basis of qualitative considerations and consider the influence on the shape of the final dilepton spectrum. Schematically, the collisional broadening that a vector meson acquires is attributed to an absorption process of the type $V+N \rightarrow R \rightarrow \pi+N$. To simplify, we approximate the 
corresponding phase space by the phase space for the process $M+m_{N} \rightarrow m_{\pi}+m_{N}$ and assume that the resonance decay proceeds through a $p$ wave. This latter freedom demonstrates again the limits of such schematic procedures. However, since this estimate is only qualitative, let us neglect for the moment these refinements.

One obtains

$$
\Gamma_{V}^{\mathrm{coll}}(M, \rho)=\Gamma_{V}^{\mathrm{coll}}\left(m_{V}, \rho\right)\left(\frac{m_{V}+m_{N}}{M+m_{N}}\right)\left(\frac{q\left(M+m_{N}, m_{N}, m_{\pi}\right)}{q\left(m_{V}+m_{N}, m_{N}, m_{\pi}\right)}\right)^{3}
$$

with

$$
q\left(M+m_{N}, m_{N}, m_{\pi}\right)=\frac{\sqrt{\left[\left(M+m_{N}\right)^{2}-\left(m_{N}+m_{\pi}\right)^{2}\right]\left[\left(M+m_{N}\right)^{2}-\left(m_{N}-m_{\pi}\right)^{2}\right]}}{2\left(M+m_{N}\right)} .
$$

As one can see, in this approximation, the vector meson threshold is shifted from $2 m_{\pi}$ to $m_{\pi}$ for the $\rho$ meson and from $3 m_{\pi}$ to $m_{\pi}$ for the $\omega$ meson. The choice affects the shape of the $\omega$ width much more than the shape of the $\rho$ width. The influence of the choice for the energy dependence of the collisional width is illustrated in Fig. 11 for the case $\rho_{B}=2 \rho_{0}$ and for $\Gamma_{\rho}^{\text {tot }}\left(\rho_{0}, m_{\rho}\right)=250$ and $\Gamma_{\omega}^{\text {tot }}\left(\rho_{0}, m_{\omega}\right)=125 \mathrm{MeV}$. In particular, for the $\omega$ meson, the shift of the threshold leads to a large enhancement of the $\omega$ width at lower invariant masses.
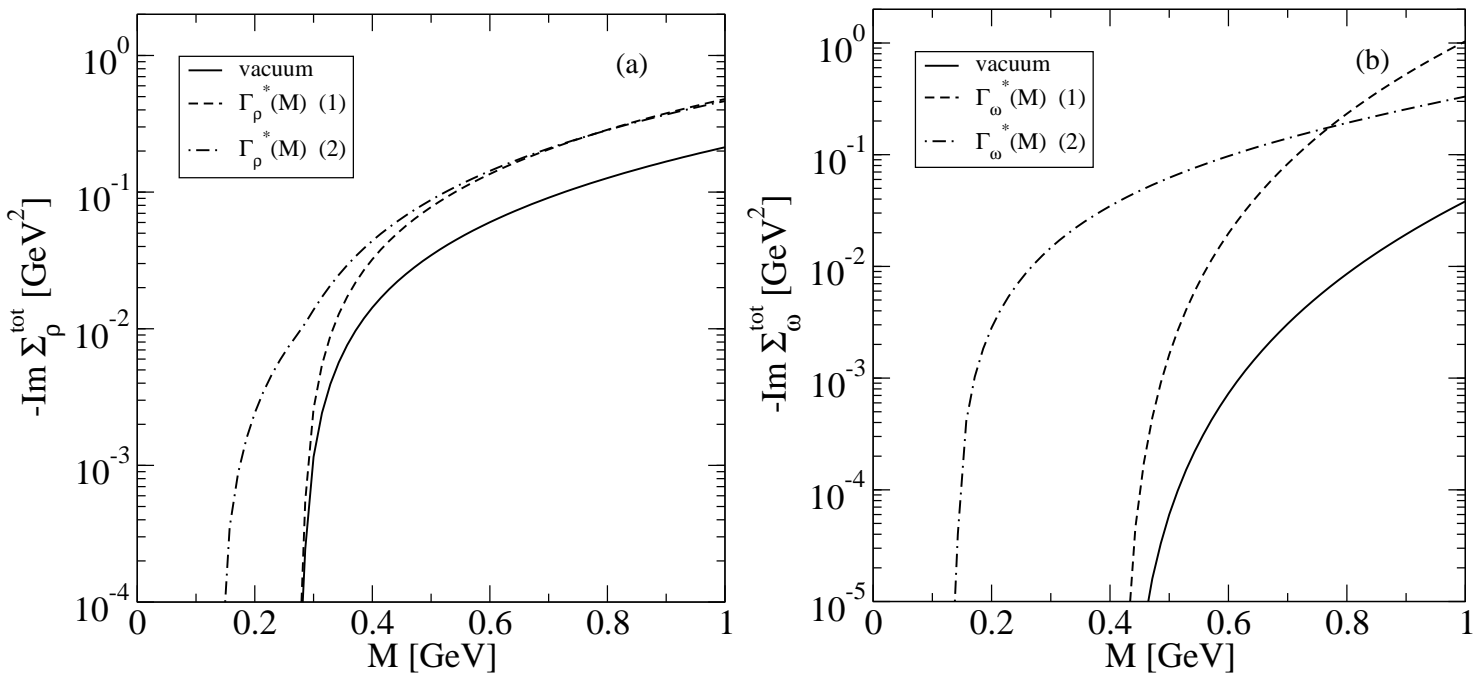

FIG. 11: (a) Imaginary part of the in-medium self-energy $-\Im \Sigma_{\rho}^{\mathrm{tot}}(\rho, M)=m_{\rho} \Gamma_{\rho}^{\mathrm{tot}}(\rho, M)$ of the $\rho$ meson in vacuum (full line) and at $\rho=2 \rho_{0}$ for $\Gamma_{\rho}^{\text {tot }}\left(\rho_{0}, m_{\rho}\right)=250 \mathrm{MeV}$ (dashed and dashed-dotted lines). (b) Imaginary part of the in-medium self-energy $-\Im \Sigma_{\omega}^{\text {tot }}(\rho, M)=m_{\omega} \Gamma_{\omega}^{\text {tot }}(\rho, M)$ of the $\omega$ meson in vacuum (full line) and at $\rho=2 \rho_{0}$ for $\Gamma_{\omega}^{\text {tot }}\left(\rho_{0}, m_{\omega}\right)=125 \mathrm{MeV}$ (dashed and dashed-dotted lines). For both panels, the dashed line corresponds to the assumption that the collisional width has the same energy dependence as the vacuum width. The dashed-dotted line corresponds to the assumption that the collisional width has the energy dependence in Eq. (26).

However, one has to keep in mind that the in-medium $\rho$ and $\omega$ widths enter into the expressions for the the covariant form factors, see Eqs. (13)-(15). Their modulus squared determines the width $\Gamma\left(R \rightarrow N \gamma^{*}\right)$ [Eq. (24) $]$. Thus, only when appreciable differences arise in the covariant form factors will the difference in the energy dependence of the in-medium vector meson width be visible in the final dilepton spectrum. Now let $\Gamma_{\rho}^{*[1]}(M)$ be the $\rho$ meson in-medium width with an energy 
dependence analogous to the vacuum width and $\Gamma_{\rho}^{*[2]}(M)$ the $\rho$ meson in-medium width with an energy dependence according to Eq. (26). Correspondingly, we set

$$
\begin{aligned}
& F_{\rho}^{[1 / 2]}=\frac{m_{\rho}^{2}}{m_{\rho}^{2}-i m_{\rho} \Gamma_{\rho}^{*[1 / 2]}(M)-M^{2}}, \\
& F_{\omega}^{[1 / 2]}=\frac{m_{\omega}^{2}}{m_{\omega}^{2}-i m_{\omega} \Gamma_{\omega}^{*[1 / 2]}(M)-M^{2}} .
\end{aligned}
$$

We refer now to the $\omega$ meson, but the same considerations are valid for the $\rho$ meson. It can be easily realized that $\left|F_{\omega}^{[2]}\right|^{2} \approx\left|F_{\omega}^{[1]}\right|^{2} \approx 1$ when $\left(m_{\omega}^{2}-M^{2}\right)^{2} \gg\left(m_{\omega} \Gamma_{\omega}^{*[i]}\right)^{2}(i=1,2)$. Thus, the mass region where sensible differences between the two cases can be found is typically restricted to the mass region around the vector meson peak. Concerning the $\rho$ meson, $\Gamma_{\rho}^{*[2]}$ and $\Gamma_{\rho}^{*[1]}$ are practically identical in the region of the vector meson peak, as can be seen from Fig,11.

Therefore, we do not expect differences between $\left|F_{\rho}^{[2]}\right|^{2}$ and $\left|F_{\rho}^{[1]}\right|^{2}$. Concerning the $\omega$ meson, $\Gamma_{\omega}^{*[2]}$ and $\Gamma_{\omega}^{*[1]}$ differ substantially in the peak region, although the main differences arise at lower masses, i.e., from slightly above $m_{\pi}$ up to slightly above $3 m_{\pi}$, because of the different thresholds. In addition, one should also consider interference terms of the form $F_{\rho}^{[i]} F_{\omega}^{[i]}$. These terms can, however, drive either a constructive or destructive interference, and therefore it is not possible to comment on their effect in general within a simple scheme.

The resulting dilepton spectra obtained for the two choices discussed above are shown in Fig. 12 Here one finds that the contributions from the $\Delta$ resonances, which couple only to the $\rho$ meson, are practically identical in the two cases. Slight differences are visible for the $N^{*}$ resonances around the vector meson peak. The differences are more evident in the case of larger values of the widths, Fig. 12(b). However, even in this case, the total spectra differ at most by a factor of 1.3 [96].
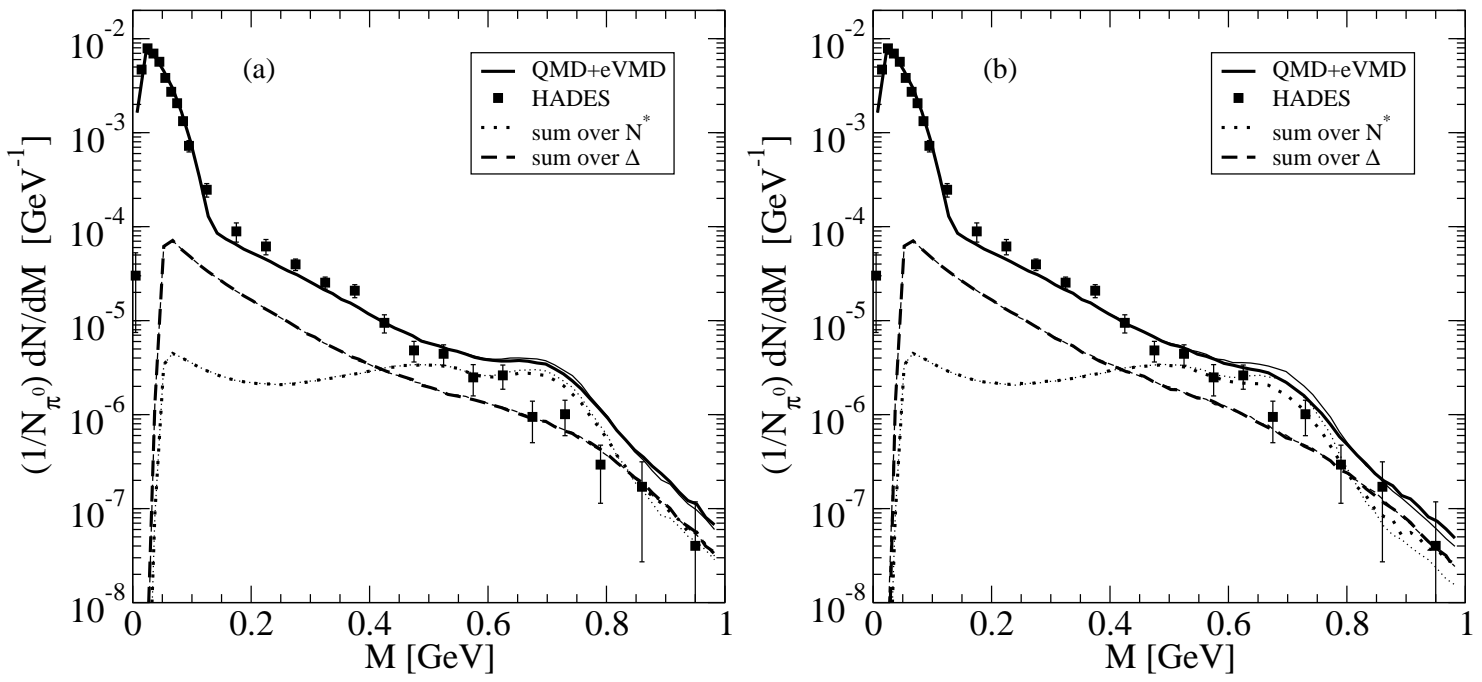

FIG. 12: Dilepton spectrum in $\mathrm{C}+\mathrm{C}$ collisions at $2.0 \mathrm{~A} \mathrm{GeV}$ for different values of the in-medium $\rho$ and $\omega$ widths and different choices for the energy dependence of the collision width. The thick lines refer to an energy dependence estimated from the $V+N \rightarrow R \rightarrow \pi+N$ as discussed in the text. The thin lines correspond to the same calculations shown in Fig. 10 and are shown for comparison. (a) $\Gamma_{\rho}^{\text {tot }}\left(\rho_{0}\right)=200$ and $\Gamma_{\omega}^{\mathrm{tot}}\left(\rho_{0}\right)=60 \mathrm{MeV}$. (b) $\Gamma_{\rho}^{\mathrm{tot}}\left(\rho_{0}\right)=250$ and $\Gamma_{\omega}^{\mathrm{tot}}\left(\rho_{0}\right)=125 \mathrm{MeV}$.

For a consistent evaluation of the energy dependence resulting from $V+N \rightarrow R \rightarrow \pi+N$ processes, one should sum up over all important resonances that couple to the $N+V$ system, 
each taken with a different weight according to their relative coupling strength, and determine for each mode the corresponding angular momentum of the $\pi N$ scattering amplitude. Moreover, the invariant mass squared of the intermediate resonance would be $s=\left(p_{N}+p\right)^{2}$ which leads to a dependence on the three-momentum $\mathbf{p}$ of the vector meson. It is then clear that such a procedure would finally be analogous to the evaluation of the full spectral functions. In fact, the $V+N \rightarrow R \rightarrow \pi+N$ channel is one of the processes consistently included in our calculation of the spectral functions, since the $N \pi$ channel is one of the channels entering in the expression of the total width of the resonance.

To conclude, already these first estimates based on the collisional broadening scenario demonstrate that the HADES data show clear evidence for a strong in-medium modification of the vector meson properties. Figure 12 demonstrates, on the other hand, that though the two different choices under discussion lead to significant deviations of the vector meson widths, particularly concerning their threshold behavior, such effects are washed out to a large extent in the final spectra. However, the same argument demands a theoretical description that is as precise as possible; i.e., realistic spectral functions should be applied rather than pushing schematic models too far.

\section{Dropping mass scenario}

However, before adopting realistic spectral functions, we want to investigate the dropping mass scenario à la Brown-Rho, which has been widely used in the literature. Thus, we performed calculations for an in-medium scenario that differs from the previous one by the additional assumption that the vector meson mass scales with density according to a $m_{V}^{*}=m_{V}\left(1-\alpha \rho_{B} / \rho_{0}\right)$ law, with $\alpha=0.2$. The results are shown in Fig. 13, where Fig. 13(a) refers to the choice $\Gamma_{\rho}^{\text {tot }}\left(\rho_{0}\right)=200 \mathrm{MeV}$ and $\Gamma_{\omega}^{\text {tot }}\left(\rho_{0}\right)=60 \mathrm{MeV}$, and Fig. 13(b) to the choice $\Gamma_{\rho}^{\text {tot }}\left(\rho_{0}\right)=250, \Gamma_{\omega}^{\text {tot }}\left(\rho_{0}\right)=125 \mathrm{MeV}$. The
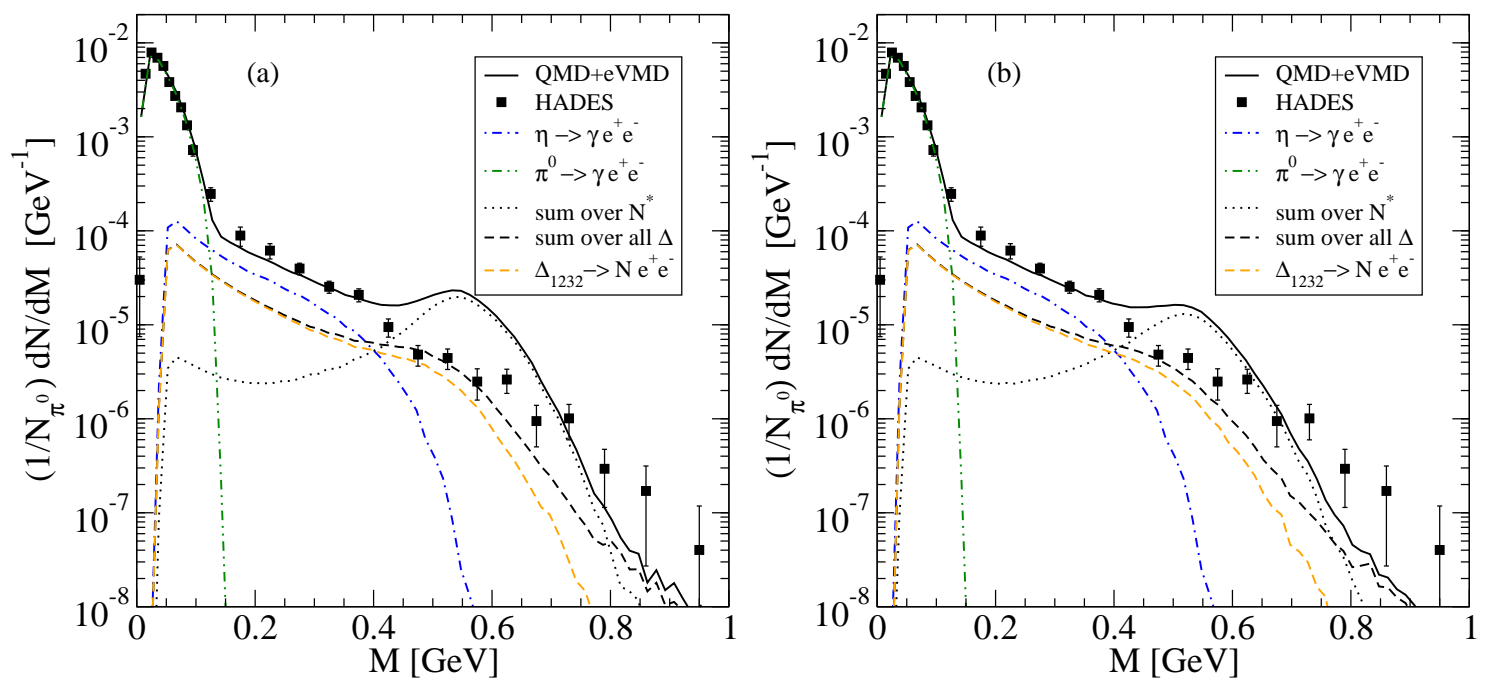

FIG. 13: (Color online) Dilepton spectrum in $\mathrm{C}+\mathrm{C}$ collisions at $2.0 \mathrm{AGeV}$ for different values of the inmedium $\rho$ and $\omega$ widths when an in-medium vector meson mass $m_{V}^{*}=m_{V}\left(1-\alpha \rho_{B} / \rho_{0}\right)$ is introduced. (a) $\Gamma_{\rho}^{\text {tot }}\left(\rho_{0}\right)=200$ and $\Gamma_{\omega}^{\text {tot }}\left(\rho_{0}\right)=60 \mathrm{MeV}$. (b) $\Gamma_{\rho}^{\text {tot }}\left(\rho_{0}\right)=250$ and $\Gamma_{\omega}^{\text {tot }}\left(\rho_{0}\right)=125 \mathrm{MeV}$.

inclusion of a dropping in-medium vector meson mass results in a global shift of the vector meson spectral strength to lower masses. Thus, the corresponding theoretical spectrum is enhanced 
at lower invariant masses resulting in a sizable overestimation of the experimental data in the $0.4 \leq M \leq 0.7 \mathrm{GeV}$ mass region. At the same time, the experimental data are underestimated in the region around and above the vector meson peak because of the lack of spectral strength around the (vacuum) vector meson peak. Note that the same underestimation of the vector meson peak was observed when adopting the dropping mass scenario to the recent high resolution CERES data 91]. The CERES analysis focused, however, only on the in-medium $\rho$ meson.

Concerning the low mass region, $m_{\pi} \leq M \leq 0.4 \mathrm{GeV}$, the presence of additional strength moves the spectrum closer to the experimental data in the mass region $M \sim 0.3-0.4 \mathrm{GeV}$. However, this region remains slightly but systematically underestimated. In summary, one can conclude that a naive Brown-Rho scaling is too schematic in order to explain the spectrum. This finding is consistent with the previous theoretical analysis of the DLS data at $1 A \mathrm{GeV}[34,35]$.

\section{In-medium spectral functions}

Let us now pass to the investigation of in-medium properties based on the in-medium selfenergies of the vector mesons calculated within NRD+eVMD. First, we present in Fig. 14(a) the dilepton spectrum obtained with "first iteration" $\rho$ and $\omega$ spectral functions, i.e., neglecting the in-medium modification of the nucleon resonance widths.
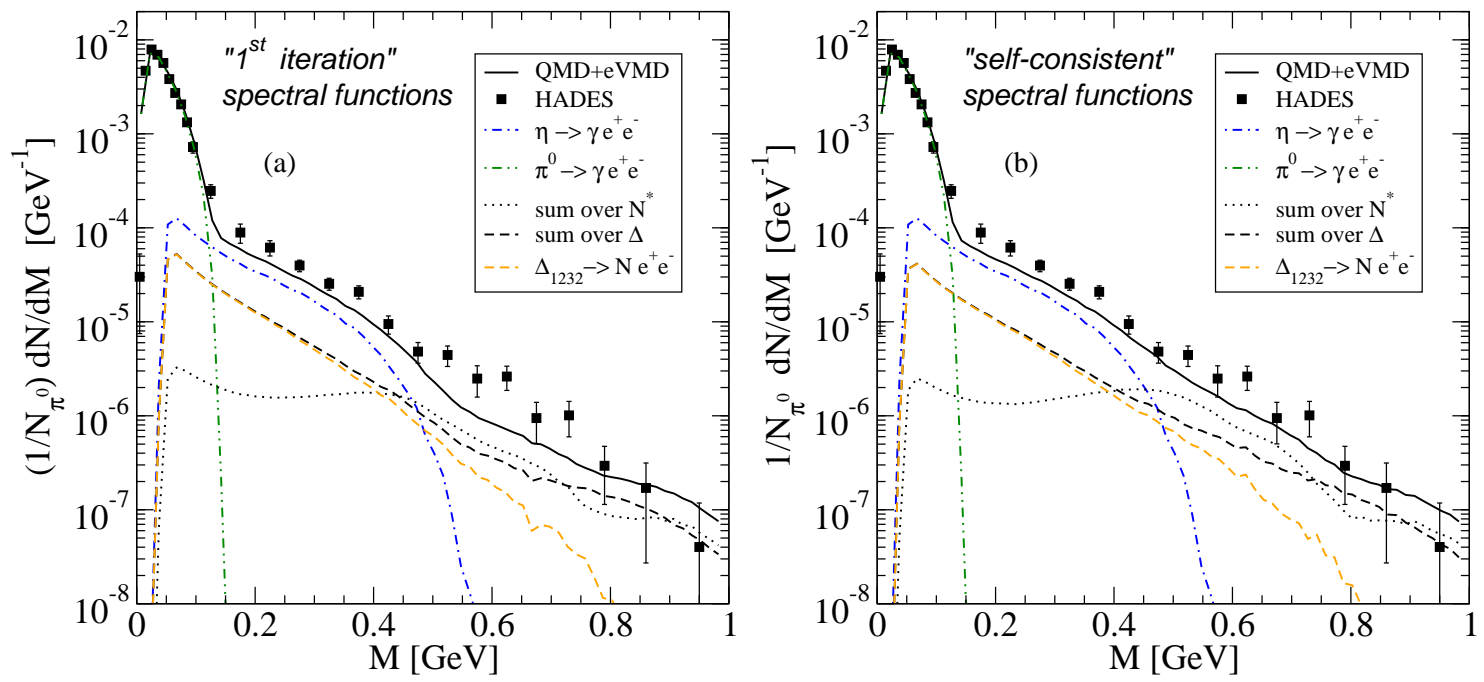

FIG. 14: (Color online) Dilepton spectrum in $\mathrm{C}+\mathrm{C}$ collisions at 2.0A GeV resulting from the inclusion of $\rho$ - and $\omega$-meson spectral functions calculated within the NRD+eVMD model. The spectral functions affect the branching ratios for the Dalitz decays of the baryon resonances, as explained in the text. (a) Inclusion of vector meson self-energies determined from vacuum nucleon resonance properties. (b) Inclusion of vector meson self-energies calculated in a self-consistent iteration scheme that takes into account the in-medium modifications of the nucleon resonance widths induced by the in-medium spectral functions of the vector mesons.

The spectral functions induce a depletion of the theoretical spectrum in the mass region $0.45 \leq$ $M \leq 0.75 \mathrm{GeV}$ which is not supported by the data. The result can be better understood with the help of Fig. 15, which shows the corresponding $\rho$ and $\omega$ contributions which enter into the nucleon resonance form factors and determine thus the dilepton production rates. The form factors are 
determined at saturation density and twice saturation density, $\rho_{B}=\rho_{0}$ and $\rho_{B}=2 \rho_{0}$, in both cases for a vector meson at rest relative to the nuclear medium (dashed lines).

The complex structure of the mesonic self-energies is clearly reflected in the form factors, which no longer preserve the simple Lorentzian-like shape typical for the vacuum. In particular, for the $\rho$ as well as for the $\omega$ we observe a strong minimum around $0.5 \lesssim M \lesssim 0.6 \mathrm{GeV}$ between two maxima at $0.4 \lesssim M \lesssim 0.5 \mathrm{GeV}$ and $M \sim 0.8 \mathrm{GeV}$.

The particular shape of the form factor is determined by the interplay of both the real and imaginary part of the self-energy. However, switching off the real part of the self-energy, we observe that the depletion of the form factor between $M \sim 0.5$ and $0.8 \mathrm{GeV}$ is mainly caused by large values of the imaginary part of the self-energy in this region. The latter is shown in Fig. 16. This increase is due to the strong coupling to specific resonances, i.e., the $N^{*}(1520)$ for the $\rho$ meson and the $N^{*}(1535)$ for the $\omega$ meson. The corresponding bump structure is a typical feature for this class of models which couple vector mesons to resonance-hole states.

The inclusion of the in-medium resonance properties caused by the vector meson spectral functions, i.e., self-consistency, reduces the imaginary part of the self-energy in this region (see Fig. 16). In the case of the $\omega$ meson, for example, the reduction at $M=0.57 \mathrm{GeV}$ is about a factor of 2.5. As a consequence, the form factors, shown in Fig. 15, are enhanced.

This has an effect on the dilepton spectrum. The spectrum obtained with self-consistent spectral functions is shown in Fig. 14(b). The inclusion of the in-medium properties of the nucleon resonances moves the theoretical spectrum closer to the experimental data in the mass region $0.45 \leq M \leq 0.75 \mathrm{GeV}$. This demonstrates the importance of higher order effects, i.e., taking inmedium modifications for the nucleon resonances into account when the vector meson properties are described by the coupling to nucleon-resonance hole states.

For the mass region $M>0.4$, we conclude that the parameter-free determination of the inmedium dilepton spectrum, performed within an approach that attempts to describe simultaneously and with the same model parameters [97] the phenomena of dilepton and vector meson production as well as their in-medium modifications, gives a reasonable description of the experimental data.

However, some data points remain still underestimated. This suggests that the NRD $+\mathrm{eVMD}$ model predicts a too strong absorption of vector mesons. One possible reason for the present underestimation of the experimental data is the use of some poorly constrained eVMD model parameters, in particular the $R N \omega$ couplings. Probably the most relevant case is the $N^{*}(1535)$ resonance, with its strong coupling to the $\omega$ meson predicted by the eVMD model though a decay of this resonance to $N \omega$ has not been measured yet. Another reason might be that the $\omega$-meson spectral function in particular is not normalized in the mass region of our interest. The violation of normalization ranges from about $30 \%$ at $\rho=\rho_{0}$ to about $45 \%$ at $\rho=2 \rho_{0}$. [98] In principle, this represents no inconsistency, since spectral functions must satisfy the sum rule in the entire invariant mass range (up to $M=\infty$ ) and not necessarily already in the finite mass interval in which we work. This effect should, however, be investigated in future work.

Regarding the low mass region, $m_{\pi} \leq M \leq 0.4 \mathrm{GeV}$, the introduction of in-medium spectral functions does not provide a solution for the underestimation of the experimental data. On the contrary, because of the finite value of the imaginary part of the self-energy at $M \sim 0$ for high vector meson three-momenta $\mathbf{p}\left[\Im \Sigma_{V}^{\text {tot }}(M=0) \neq 0\right.$ for $\left.\mathbf{p} \neq 0\right]$, at high momenta we have $\left|F_{V}(M=0)\right|^{2}<1$ with a consequent reduction of strength. We can therefore conclude that for the explanation of the low mass region, one has to take into account additional effects and/or sources. 

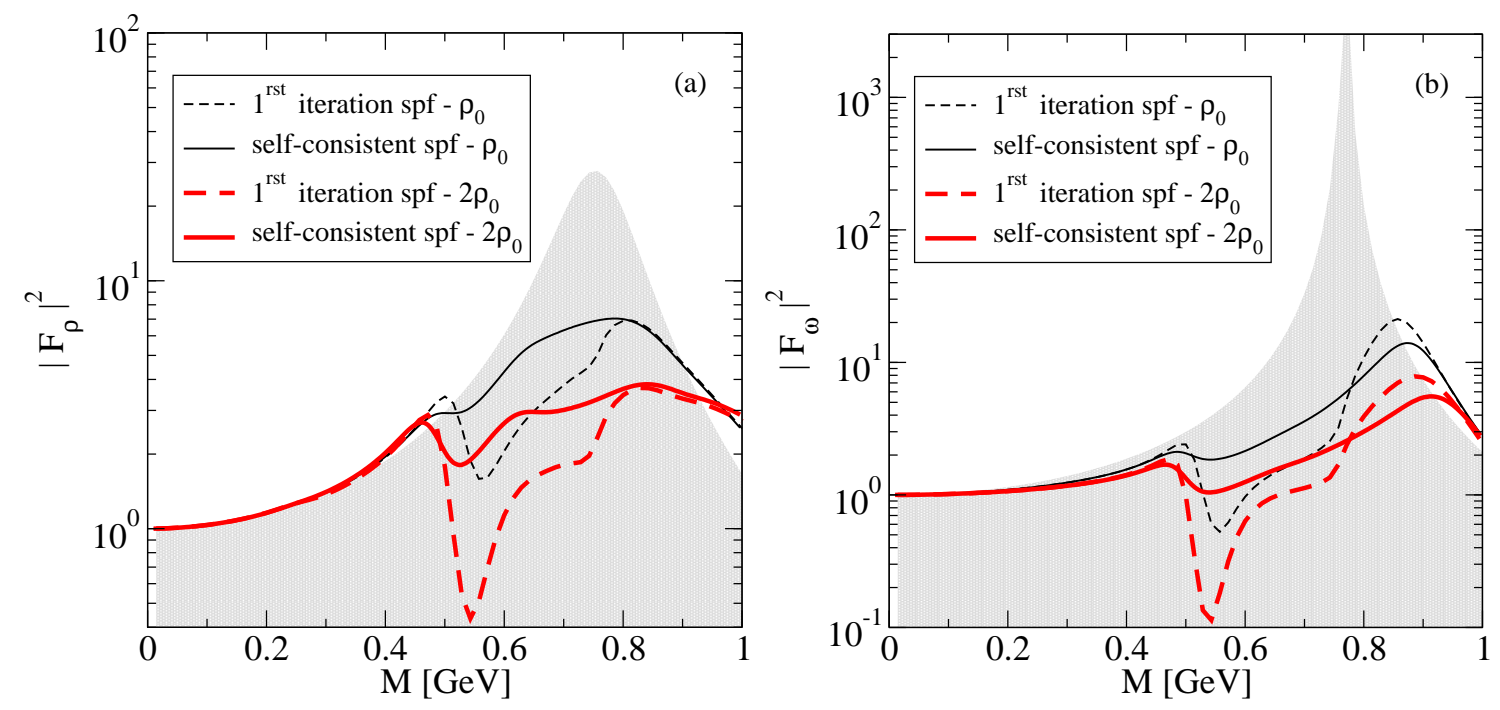

FIG. 15: (Color online) (a) Modulus squared of the $\rho$ meson contribution to the covariant form factor $\left|F_{\rho}\right|^{2}$ at $\rho_{B}=\rho_{0}$ (thin lines) and $\rho_{B}=2 \rho_{0}$ (thick lines). (b) Modulus squared of the $\omega$ meson contribution to the covariant form factor $\left|F_{\omega}\right|^{2}$ at $\rho_{B}=\rho_{0}$ (thin lines) and $\rho_{B}=2 \rho_{0}$ (thick lines). For both panels the dashed lines correspond to vector meson self-energies calculated from vacuum nucleon resonance properties. The full lines correspond to vector meson self-energies calculated in a self-consistent iteration scheme that takes into account the in-medium modifications of the nucleon resonance widths induced by the in-medium spectral functions of the vector mesons. Shaded areas indicate the vacuum form factors.
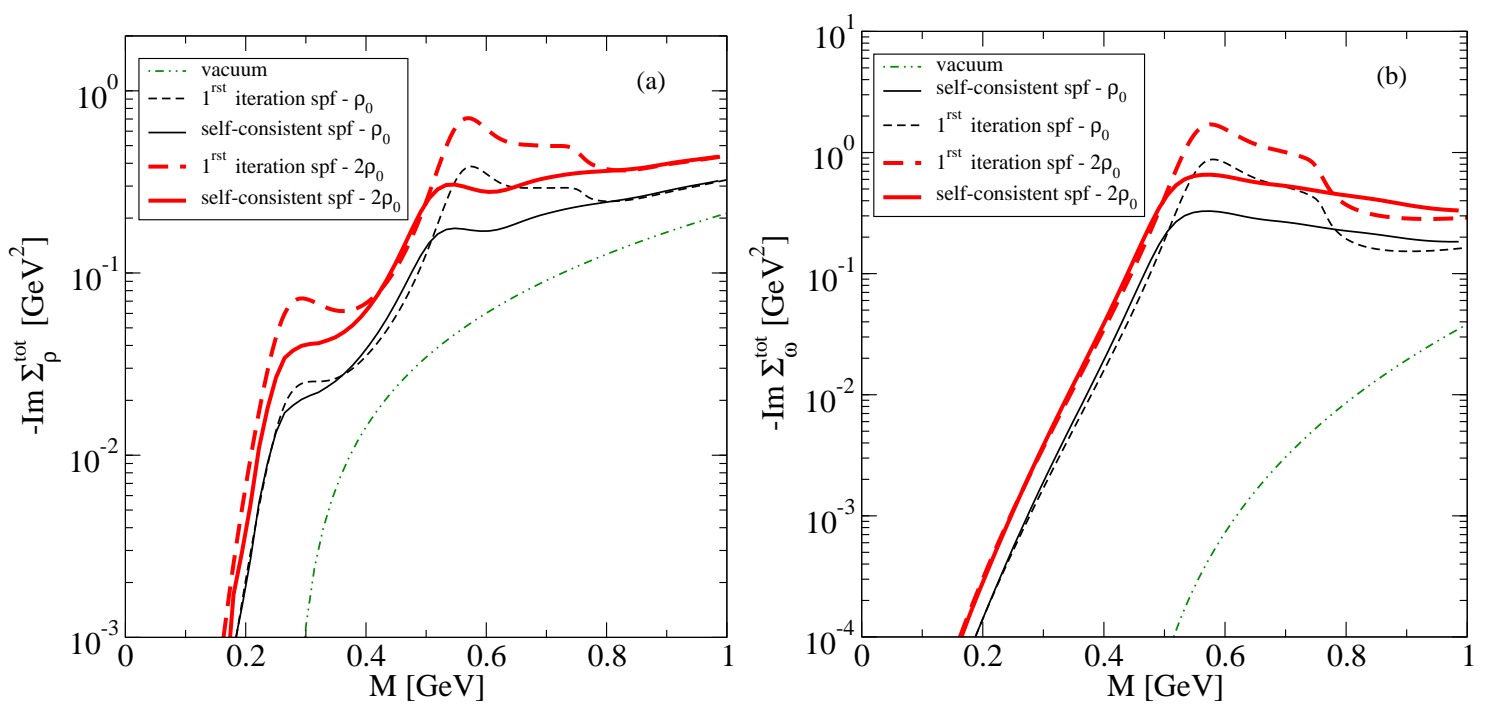

FIG. 16: (Color online) Imaginary part of the in-medium self-energy of the (a) $\rho$ meson and (b) $\omega$ meson, in vacuum (dashed-double-dotted lines), at $\rho_{B}=\rho_{0}$ (thin lines) and $\rho_{B}=2 \rho_{0}$ (thick lines). Dashed lines correspond to vector meson self-energies calculated from vacuum nucleon resonance properties. Full lines correspond to vector meson self-energies calculated in a self-consistent iteration scheme that takes into account the in-medium modifications of the nucleon resonance widths induced by the in-medium spectral functions of the vector mesons. 


\section{CONCLUSIONS}

We determined the modification of the $\rho$ and $\omega$ meson properties in nuclear matter within a resonance model and investigated the nonresonant contributions to the vector meson self-energy. For both vector mesons, we found a substantial broadening of the width and a significant shift of spectral strength down to smaller invariant masses. In particular at small momenta, the coupling of the $\rho$ meson to the $N^{*}(1520) N^{-1}$ state and that of the $\omega$ meson to the $N^{*}(1535) N^{-1}$ state lead to pronounced double-peak structures in the spectral functions. In a first approximation, the spectral functions were determined from vacuum nucleon resonance properties. Going beyond this approximation, the in-medium modification of the nucleon resonance widths induced by the modified $\rho$ and $\omega$ mesons has been included. This leads to a self-consistent calculation of the vector meson spectral functions, which mainly reduces the peaks due to the coupling to $N^{*}(1520) N^{-1}$ and $N^{*}(1535) N^{-1}$ states.

In a next step, we investigated the influence of different in-medium scenarios for the vector mesons on the dilepton production rate in heavy-ion collisions. The dilepton spectrum has been calculated exemplarily for the reaction $\mathrm{C}+\mathrm{C}$ at $2.0 \mathrm{~A} \mathrm{GeV}$ for which experimental data have been recently released by the HADES Collaboration.

Already the estimates based on a schematic collisional broadening scenario, i.e., the comparison with data, support strong in-medium modification of the vector meson properties. In the dropping mass scenario, we found, even when taking additionally into account the collisional broadening of the vector meson widths, that the dilepton spectrum overestimates the experimental data at invariant masses below the vector meson peak and underestimates them in the region around and above the peak.

Finally, we went beyond the schematic inclusion of in-medium effects and included the vector meson properties consistently, i.e., in terms of the in-medium self-energies microscopically calculated within our model. We found that self-energies determined from vacuum nucleon resonance properties give a poor description of the experimental data in the invariant mass region $0.45 \leq M \leq 0.75 \mathrm{GeV}$. On the contrary, the self-consistent iteration scheme provides a reasonable description of the data in the same mass region. This demonstrates the importance of consistent inclusion of in-medium properties.

However, for the low mass region $\left(m_{\pi} \leq M \leq 0.4 \mathrm{GeV}\right)$ we found that the inclusion of $\rho$ and $\omega$ spectral functions does not improve the theoretical description of the dilepton spectrum and experimental data remain slightly underestimated.

In summary, the investigation represents a first step toward a unified understanding of dilepton spectra and vector meson properties in heavy-ion collisions at intermediate energies. The same model and the same set of parameters were used to describe the interconnected phenomena of dilepton and vector meson production and their in-medium modifications. Forthcoming data, from elementary reactions as well as from heavy-ion collisions of heavy systems, will certainly help to further reduce still existing model uncertainties. An extension of the present approach to finite baryon chemical potential and temperature would further allow one to test the spectral properties beyond HADES conditions, e.g., by a comparision with the NA60 data. 


\section{Acknowledgments}

We are grateful to the HADES Collaboration for help concerning the HADES filter program and for providing us with the experimental data. This work was supported by the European Graduate School Basel-Graz-Tübingen and by the RFBR Grant No. 06-02-04004 and the DFG Grant No. 436 RUS 113/72/0-2.

\section{APPENDIX A: GAUGE INVARIANCE IN EVMD}

The VMD model and its modifications introduce the mixing of a photon with vector mesons $\rho^{0}, \omega, \phi$, etc. Such a mixing can, in principle, generate finite photon masses and destroy gauge invariance. This problem has been solved for the VMD model by Kroll, Lee and Zumomino 92] constructing an effective Lagrangian for photons and vector mesons which reproduces the VMD predictions. We present first a distinct consistency proof and then show how the method [92] can be generalized to the eVMD model.

\section{Final-state interaction (FSI) method}

We start from an effective Lagrangian involving pions interacting with photons. An example of such a Lagrangian is the nonlinear $\sigma$ model and, more generally, the chiral perturbation theory (ChPT) to a fixed order of the loop expansion. The vector mesons appear as resonances in the two-pion scattering channel ( $\rho$ mesons) and the three-meson scattering channel ( $\omega$ mesons).

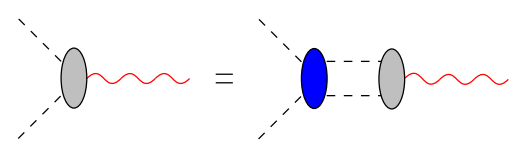

FIG. 17: (Color online) Diagrammatic representation of the FSI of pions (dashed lines) contributing to the form factor in the $\rho$-meson channel. The photon line is shown as a wavy line.

Let us consider an absorption of a photon in an isovector channel, as shown in Fig. 17. Applying two-body unitarity and taking into account analyticity (see, e.g., Ref. [93], Chap. 18), we replace the point-like vertex $e$ by $e P_{l}(t) / D_{J}(t)$ where $t=q^{2}$ is the photon momentum squared, $P_{l}(t)$ is a polynomial of the degree $l$, and $D_{J}(t)$ is the Jost function defined in terms of the $p$-wave isovector two-pion scattering phase shift $\delta(t)$ :

$$
D_{J}(t)=\exp \left[-\frac{t}{\pi} \int_{t_{0}}^{\infty} \frac{\delta\left(t^{\prime}\right) d t^{\prime}}{t^{\prime}\left(t-t^{\prime}\right)}\right]
$$

where $t_{0}$ is the two-pion threshold.

In the no-width approximation, the phase shift accounting for the existence of $n$ resonances is given by

$$
\delta(t)=\sum_{k=1}^{n} \pi \theta\left(t-m_{k}^{2}\right)
$$


where $m_{k}$ is the mass of the $k$ th radial excitation of the $\rho^{0}$ meson. Substituting this expression into Eq.(A1), we obtain

$$
F(t)=P_{l}(t) \prod_{k=1}^{n} \frac{m_{k}^{2}}{t-m_{k}^{2}}
$$

The requirement $F(t) \rightarrow 0$ at $t \rightarrow \infty$ gives $l<n$.

Analytical functions are fixed by their singularities. The representation (A3) can be rewritten in an equivalent additive form

$$
F(t)=\sum_{k=1}^{n} c^{k} \frac{m_{k}^{2}}{m_{k}^{2}-t}
$$

where $c^{k}$ are some coefficients. The normalization condition $F(0)=1$ and the quark counting rules impose constraints for $c^{k}$.

The effective pion Lagrangian is well defined, since pions are stable particles which exist as asymptotic states. In the approach presented above, the problem of gauge invariance does not appear, since gauge invariance of the effective Lagrangian ensures a transverse polarization tensor of photons and the vanishing photon mass. The vector mesons are resonances accounted for by the the final-state interactions.

\section{Effective Lagrangian method}

The vector mesons are unstable particles and do not exist as asymptotic states. Nevertheless, the effective Lagrangian method is useful in formulating vector meson effective interactions. Kroll, Lee, and Zumomino [92] proposed an effective Lagrangian for the VMD model to illustrate its gauge invariance. We extend their arguments for a family of $n \rho^{0}$ mesons interacting with photons. An effective Lagrangian can be written as

$$
\mathcal{L}_{\text {eff }}=-\frac{1}{4} F_{\mu \nu} F_{\mu \nu}+\sum_{k=1}^{n}\left(-\frac{1}{4} G_{\mu \nu}^{k} G_{\mu \nu}^{k}+\frac{1}{2} m_{k}^{2} B_{\mu}^{k} B_{\mu}^{k}+\frac{e}{2 g^{k}} G_{\mu \nu}^{k} F_{\mu \nu}\right)-\left(e A_{\mu}+\sum_{k=1}^{n} h^{k} B_{\mu}^{k}\right) J_{\mu},
$$

where $A_{\mu}$ is the electromagnetic vector potential, $B_{\mu}^{k}$ is the $k$ th $\rho^{0}$-meson vector potential, $J_{\mu}$ is a hadron conserved current, and $F_{\mu \nu}=\partial_{\nu} A_{\mu}-\partial_{\mu} A_{\nu}$ and $G_{\mu \nu}^{k}=\partial_{\nu} B_{\mu}^{k}-\partial_{\mu} B_{\nu}^{k}$.

Lagrangian (A5) is gauge invariant with respect to gauge transformations of the electromagnetic vector potential, so the photon interactions with the vector mesons do not violate gauge invariance and, in particular, do not generate a photon mass.

It remains to be shown that the coupling constants $g^{k}$ and $h^{k}$ can be chosen such that they reproduce the eVMD predictions. For each vector meson, we consider the two diagrams shown on Fig. 18. Their sum gives

$$
F(t)=1+\sum_{k=1}^{n} \frac{1}{g^{k}} \frac{t}{m_{k}^{2}-t} h^{k}
$$

The spectral functions of the form factors and their asymptotic behavior depend on the type of transition. The usual VMD appears for $n=1$. It corresponds to asymptotics $F(t) \sim 1 / t$ at $t \rightarrow \infty$. 
If we set $h^{1}=g^{1}$, the monopole form factor is reproduced:

$$
F(t)=\frac{m_{1}^{2}}{m_{1}^{2}-t}
$$

For $m_{1}=m_{\rho}$ it describes well the pion form factor in the space-like region. The model [94, 95] of the pion form factor, which represents an improvement of the VMD to account for the analyticity and two-body unitarity of the pion form factor, and the $\rho$-meson width, works well in both the space- and time-like regions.

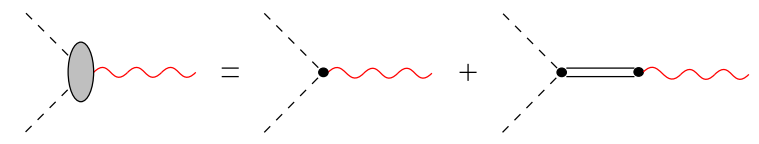

FIG. 18: (Color online) Diagrammatic representation of the photon interaction with the electromagnetic current using the effective Lagrangian couplings. The first diagram shows the direct photon coupling and the second one shows the coupling through the family of $n \rho$ mesons (double solid line). The photon line is shown as a wavy line.

In the case of eVMD, we set $h^{k}=c^{k} g^{k}$, where $c^{k}$ are some coefficients. The form factor $F(t)$ should decay at infinity, so we obtain

$$
\sum_{k=1}^{n} c^{k}=1
$$

Then the usual representation (A4) of the eVMD form factors follows.

The quark counting rules can be satisfied selecting the coefficients $c^{k} . F(t) \sim 1 / t^{2}$ gives

$$
\sum_{k=1}^{n} c^{k} m_{k}^{2}=0
$$

Equations (A8) and (A9) have a unique solution for $n=2 . F(t) \sim 1 / t^{3}$ requires the existence of at least $n=3$ vector mesons and so on. 
[1] E. van Dalen, C. Fuchs, A. Faessler, Nucl. Phys. A 744, 227 (2004); Phys. Rev. Lett. 95, 022302 (2005).

[2] N. Bianchi et al., Phys. Lett. B 309 (1993) 5; Phys. Lett. B 325, 333 (1994).

[3] B. Krusche, J. Ahrens, R. Beck, I. J. D. MacGregor, J. C. McGeorge, V. Metag and H. Stroher, Phys. Rev. Lett. 86, 4764 (2001).

[4] L. A. Kondratyuk, M. I. Krivoruchenko, N. Bianchi, E. De Sanctis, V. Muccifora, Nucl. Phys. A579, 453 (1994); M. M. Giannini, E. Santopinto, Phys. Rev. C49, 1258 (1994).

[5] G.E. Brown and M. Rho, Phys. Rev. Lett. 66, 2720 (1991); Phys. Rep. 269, 333 (1996).

[6] T. Hatsuda and S.H. Lee, Phys. Rev. C 46, R34 (1992);

T. Hatsuda, S. H. Lee and H. Shiomi, Phys. Rev. C 52, 3364 (1995).

[7] S. Leuopold, Phys. Rev. C 64, 015202 (2001).

[8] S. Zschocke, O. P. Pavlenko and B. Kampfer, Phys. Lett. B 562, 57 (2003).

[9] R. Thomas, S. Zschocke and B. Kampfer, Phys. Rev. Lett. 95, 232301 (2005).

[10] F. Klingl, N. Kaiser and W. Weise, Nucl. Phys. A 624, 527 (1997).

[11] L. A. Kondratyuk, A. Sibirtsev, W. Cassing, Y. S. Golubeva and M. Effenberger, Phys. Rev. C 58 (1998) 1078.

[12] V. L. Eletsky and J. I. Kapusta, Phys. Rev. C 59 (1999) 2757.

[13] V. L. Eletsky, M. Belkacem, P. J. Ellis and J. I. Kapusta, Phys. Rev. C 64, 035202 (2001).

[14] M. Hermann, B. Friman, and W. Nörenberg, Nucl. Phys. A 560, 411 (1993); G. Chanfray and P. Schuck, Nucl. Phys. A 545, 271c (1992).

[15] F. Klingl, N. Kaiser and W. Weise, Z. Phys. A 356, 193 (1996).

[16] B. Friman and H.J. Pirner, Nucl. Phys. A 617, 496 (1997).

[17] M. Urban, M. Buballa, R. Rapp, and J. Wambach, Nucl. Phys. A 641, 433 (1998); Nucl. Phys. A 673, 357 (2000).

[18] R. Rapp and J. Wambach, Adv. Nucl. Phys. 25, 1 (2000).

[19] M. F. M. Lutz, G. Wolf and B. Friman, Nucl. Phys. A 706, 431 (2002) [Erratum-ibid. A 765, 431 (2006)].

[20] M. Post and U. Mosel, Nucl. Phys. A 688, 808 (2001).

[21] M. Post, S. Leupold and U. Mosel, Nucl. Phys. A 741, 81 (2004).

[22] V. Shklyar, H. Lenske, U. Mosel and G. Penner, Phys. Rev. C 71, 055206 (2005) [Erratum-ibid. C 72, 019903 (2005)].

[23] P. Muehlich, V. Shklyar, S. Leupold, U. Mosel and M. Post, Nucl. Phys. A 780, 187 (2006).

[24] S. A. Chin, Annals Phys. 108 (1977) 301.

[25] V. Bernard and U. G. Meissner, Nucl. Phys. A 489, 647 (1988).

[26] G. Agakichiev et al. [CERES Collaboration], Phys. Rev. Lett. 75, 1272 (1995).

[27] A. Marin et al. [CERES Collaboration], J. Phys. G 30, S709 (2004).

[28] M.A. Mazzoni, Nucl. Phys. A 566, 95c (1994); M. Masera, Nucl. Phys. A 590, 93c (1995).

[29] G. Q. Li, C. M. Ko and G. E. Brown, Phys. Rev. Lett. 75, 4007 (1995).

[30] R. Arnaldi et al. [NA60 Collaboration], Phys. Rev. Lett. 96 (2006) 162302.

[31] H. van Hees and R. Rapp, Phys. Rev. Lett. 97, 102301 (2006).

[32] R.J. Porter et al. [DLS Collaboration], Phys. Rev. Lett. 79, 1229 (1997).

[33] W.K. Wilson et al. [DLS Collaboration], Phys. Rev. C57 (1998) 1865.

[34] E. L. Bratkovskaya, W. Cassing, R. Rapp and J. Wambach, Nucl. Phys. A 634, 168 (1998).

[35] C. Ernst, S.A. Bass, M. Belkacem, H. Stocker, and W. Greiner, Phys. Rev. C 58, 447 (1998).

[36] K. Shekter, C. Fuchs, A. Faessler, M. Krivoruchenko, and B. Martemyanov, Phys. Rev. C 68, 014904 (2003).

[37] T. Eberl et al., Nucl. Phys. A 752, 433 (2005).

[38] G. Agakichiev et al. [HADES Collaboration], Phys. Rev. Lett. 98, 052302 (2007).

[39] I. Frohlich et al. [HADES Collaboration], Eur. Phys. J. A 31, 831 (2007). 
[40] D. Trnka et al. [CBELSA/TAPS Collaboration], Phys. Rev. Lett. 94, 192303 (2005).

[41] K. Ozawa et al., Phys. Rev. Lett. 86 (2001) 5019.

[42] M. Naruki et al., Phys. Rev. Lett. 96, 092301 (2006).

[43] E. L. Bratkovskaya, Phys. Lett. B 529, 26 (2002).

[44] L. P. Kaptari and B. Kampfer, Nucl. Phys. A 764, 338 (2006).

[45] E. L. Bratkovskaya and W. Cassing, Nucl. Phys. A 807, 214 (2008).

[46] M. I. Krivoruchenko, B. V. Martemyanov, A. Faessler and C. Fuchs, Annals Phys. 296, 299 (2002).

[47] A. Faessler, C. Fuchs, M. I. Krivoruchenko and B. V. Martemyanov, J. Phys. G 29, 603 (2003).

[48] S. Teis, W. Cassing, M. Effenberger, A. Hombach, U. Mosel and G. Wolf, Z. Phys. A 359, 297 (1997).

[49] A. Faessler, C. Fuchs, and M.I. Krivoruchenko, Phys. Rev. C 61, 035206 (2000).

[50] V. A. Matveev, R. M. Muradian and A. N. Tavkhelidze, Lett. Nuovo Cim. 7, 719 (1973); S. J. Brodsky and G. R. Farrar, Phys. Rev. Lett. 31, 1153 (1973); S. J. Brodsky and G. R. Farrar, Phys. Rev. D 11, 1309 (1975) ; A. I. Vainstein and V. I. Zakharov, Phys. Lett. B 72, 368 (1978).

[51] C. Fuchs, M. I. Krivoruchenko, H. L. Yadav, Amand Faessler, B. V. Martemyanov and K. Shekhter., Phys. Rev. C 67, 025202 (2003).

[52] Amand Faessler, C. Fuchs, M. I. Krivoruchenko, B. V. Martemyanov, Phys. Rev. C 68, 068201 (2003).

[53] A. Faessler, C. Fuchs, M. Krivoruchenko, and B. Martemyanov, Phys. Rev. C 70, 035211 (2004).

[54] F. Balestra et al. [DISTO Collaboration], Phys. Rev. Lett. 81, 4572 (1998).

[55] S. Abd El-Samad et al. [COSY-TOF Collaboration], Phys. Lett. B 522, 16 (2001).

[56] W.S. Uma Maheswari, C. Fuchs, A. Faessler, L. Sehn, D. Kosov, and Z. Wang, Nucl. Phys. A 628, 669 (1998).

[57] C. Fuchs, Prog. Part. Nucl. Phys. 56, 1 (2006).

[58] M. D. Cozma, C. Fuchs, E. Santini and A. Fassler, Phys. Lett. B 640, 170 (2006).

[59] G. G. Penner and U. Mosel, Phys. Rev. C 65, 055202 (2002) [Erratum-ibid. C 65, 059901 (2002)]; G. Penner and U. Mosel, Phys. Rev. C 66, 055211 (2002).

[60] D. Cabrera, E. Oset and M. J. Vicente Vacas, Nucl. Phys. A 705, 90 (2002).

[61] M. Gell-Mann, D. Sharp, G.W. Wagner, Phys. Rev. Lett 8, 261 (1962).

[62] D. M. Manley and E. M. Saleski, Phys. Rev. D 45, 4002 (1992).

[63] T. Feuster and U. Mosel, Phys. Rev. C 58, 457 (1998).

[64] T. P. Vrana, S. A. Dytman and T. S. H. Lee, Phys. Rept. 328, 181 (2000).

[65] P. D. B. Collins, An Introduction to Regge Theory And High-Energy Physics, Cambridge University Press, Cambridge, 1977.

[66] R. Machleidt, Phys. Rev. C 63, 024001 (2001).

[67] Particle Data Group, Phys. Rev. D 54, 1 (1996).

[68] F. Hibou et al., Phys. Rev. Lett. 83, 492 (1999).

[69] S. Barsov et al., Eur. Phys. J. A 31, 95 (2007).

[70] W. Peters, M. Post, H. Lenske, S. Leupold and U. Mosel, Nucl. Phys. A 632, 109 (1998).

[71] M. Djalali Chaden, Quark Matter 2006.

[72] H. W. Barz, B. Kampfer, G. Wolf and M. Zetenyi, arXiv:nucl-th/0605036.

[73] S. Leupold, Nucl. Phys. A 695, 377 (2001).

[74] W. Cassing, S. Juchem, Nucl. Phys. A 665377 (2000); Nucl. Phys. A 677445 (2000).

[75] J. Aichelin, Phys. Rept. 202, 233 (1991).

[76] C. Fuchs and T. Gaitanos, Nucl. Phys. A 714, 643 (2003).

[77] B. Schenke and C. Greiner, Phys. Rev. Lett. 98, 022301 (2007).

[78] C. Fuchs, P. Essler, T. Gaitanos and H. H. Wolter, Nucl. Phys. A 626, 987 (1997).

[79] L. V. Bravina et al., Phys. Rev. C 78, 014907 (2008).

[80] S.J. Brodsky and G.R. Farrar, Phys. Rev. Lett. 31, 1153 (1973); S.J. Brodsky and G.R. Farrar, Phys. Rev. D 11, 1309 (1975).

[81] G. Q. Li, C. M. Ko, G. E. Brown and H. Sorge, Nucl. Phys. A 611, 539 (1996).

[82] R. Shyam and U. Mosel, Phys. Rev. C 67, 065202 (2003). 
[83] C. Sturm et al. [KAOS Collaboration], Phys. Rev. Lett. 86, 39 (2001).

[84] T. Inoue and E. Oset, Nucl. Phys. A 710, 354 (2002).

[85] G. Agakishiev et al. [HADES Collaboration], Phys. Lett. B 663, 43 (2008).

[86] C. Fuchs, A. Faessler, E. Zabrodin and Y. M. Zheng, Phys. Rev. Lett. 86, 1974 (2001).

[87] R. Averbeck et al. [TAPS Collaboration], Z. Phys. A 359, 65 (1997).

[88] M. Thomere, C. Hartnack, G. Wolf and J. Aichelin, Phys. Rev. C 75, 064902 (2007).

[89] D. Schumacher, S. Vogel and M. Bleicher, Acta Phys. Hung. A 27, 451 (2006).

[90] M. I. Krivoruchenko and A. Faessler, Phys. Rev. D 65, 017502 (2002).

[91] D. Adamova et al., arXiv:nucl-ex/0611022.

[92] N. M. Kroll, T. D. Lee and B. Zumino, Phys. Rev. 157, 1376 (1967).

[93] J. D. Bjorken and S. D. Drell, Relativistic Quantum Fields, New York, McGraw-Hill (1965).

[94] W. R. Frazer and J. Fulco, Phys. Rev. Lett. 2, 365 (1959); Phys. Rev. 117, 1603 (1960); Phys. Rev. 117, 1609 (1960).

[95] G. Gounaris and J. J. Sakurai, Phys. Rev. Lett. 21, 244 (1968).

[96] Here we refer to the maximum value of the ratio of the two spectra.

[97] This statement refers to the resonance+eVMD model parameters. The additional parameters of the RQMD transport model (cross sections, potentials, etc.) have not been changed.

[98] Integral evaluated in the mass region up to $1.5 \mathrm{GeV}$. 